\title{
Homopolymer tract organization in the human malarial parasite Plasmodium falciparum and related Apicomplexan parasites
}

\author{
Karen Russell ${ }^{1}$, Chia-Ho Cheng ${ }^{2,8}$, Jeffrey W Bizzaro ${ }^{3}$, Nadia Ponts ${ }^{4}$, Richard D Emes ${ }^{5,6}$, Karine Le Roch?
} Kenneth A Marx ${ }^{2}$ and Paul Horrocks ${ }^{1 *}$

\begin{abstract}
Background: Homopolymeric tracts, particularly poly dA.dT, are enriched within the intergenic sequences of eukaryotic genomes where they appear to act as intrinsic regulators of nucleosome positioning. A previous study of the incomplete genome of the human malarial parasite Plasmodium falciparum reports a higher than expected enrichment of poly dA.dT tracts, far above that anticipated even in this highly AT rich genome. Here we report an analysis of the relative frequency, length and spatial arrangement of homopolymer tracts for the complete $P$. falciparum genome, extending this analysis to twelve additional genomes of Apicomplexan parasites important to human and animal health. In addition, using nucleosome-positioning data available for $P$. falciparum, we explore the correlation of poly dA.dT tracts with nucleosome-positioning data over key expression landmarks within intergenic regions.

Results: We describe three apparent lineage-specific patterns of homopolymeric tract organization within the intergenic regions of these Apicomplexan parasites. Moreover, a striking pattern of enrichment of overly long poly $\mathrm{dA}$.dT tracts in the intergenic regions of Plasmodium spp. uniquely extends into protein coding sequences. There is a conserved spatial arrangement of poly dA.dT immediately flanking open reading frames and over predicted core promoter sites. These key landmarks are all relatively depleted in nucleosomes in $P$. falciparum, as would be expected for poly dA.dT acting as nucleosome exclusion sequences.
\end{abstract}

Conclusions: Previous comparative studies of homopolymer tract organization emphasize evolutionary diversity; this is the first report of such an analysis within a single phylum. Our data provide insights into the evolution of homopolymeric tracts and the selective pressures at play in their maintenance and expansion.

Keywords: Poly dA.dT, Intergenic regions, Malaria, Nucleosome, Gene expression

\section{Background}

Genome-wide surveys of homopolymer tract frequencies in eukaryotic genomes reveal a striking enrichment of long poly dA.dT tracts in intergenic regions (IGR) compared to expected frequencies of random tracts of equivalent base composition [1-3]. Utilizing techniques such as MAINEFAIRE (micrococcal nuclease-assisted isolation of nucleosomal elements and formaldehyde assisted isolation of regulatory elements) or chromatin immunoprecipitation, both coupled to high throughput sequencing approaches,

\footnotetext{
* Correspondence: p.d.horrocks@keele.ac.uk

${ }^{1}$ Institute for Science and Technology in Medicine, Keele University, Stoke-on-Trent ST5 5BG, Staffordshire, UK

Full list of author information is available at the end of the article
}

reveal a relative depletion of nucleosomes over poly dA.dT tracts of increasing length [2,4-6]. The shorter helical repeat distance within poly dA.dT tracts, along with a narrower and deeper minor grove with a defined spine of hydration, appear to energetically disfavour the necessary remodeling of these tracts for nucleosome binding (for review see [7]). Over recent years, high-resolution maps of nucleosome positioning in a number of eukaryotes have revealed that these poly dA.dT tracts represent a canonical feature of an intrinsic nucleosome positioning code within DNA sequences [4,5,8-11]. A second canonical feature, a $10 \mathrm{bp}$ periodicity of AA/TT/TA dinucleotide repeats, provides an opposing role by promoting sharp bending of DNA necessary for wrapping around nucleosomes. Thus, the 
absence of dinucleotide repeats, together with the presence tracts of poly dA.dT, provides for an ordered, and modifiable, nucleosome landscape where nucleosome depleted regions (NDR) typically act as barriers between well-ordered arrays of nucleosomes organized over exonic sequences $[5,7,9,10]$. Critically, these flanking 5' and 3' NDR, located in the immediate IGR, provide key sites for the regulation of gene expression. 5' NFR facilitating access of specific transcription factors and the basal transcriptional apparatus to the genomic template, with the more recently described 3' NDR offering a region for RNA polymerase II (RNAPolII) complex disassembly over transcription termination sites as well as a site for initiation of antisense transcription [4,11].

Analysis of chromosomes 2 and 3 of Plasmodium falciparum, the aetiological agent of the most virulent form of human malaria, reveals higher than expected frequencies of over-long polydA.dT tracts in IGR [1,3]. Whilst few studies have explored the function of poly dA.dT tracts in the control of gene expression in this parasite, those that have support a role for these tracts in altering absolute levels of gene expression [12,13]. Genome-wide profiling of nucleosome positioning during the intraerythrocytic (IE) stage of development indicates variation in positioning subject to temporal developmentally-linked control [14-16]. Maximal levels of nucleosome occupancy are apparent in the final stages of IE development with minimum nucleosome occupancy levels coincident with $\mathrm{S}$-phase and the highest levels of transcriptional activity [16]. P. falciparum is unusual in that the majority of its genome is maintained in a euchromatic state, with no evidence of the highest orders of chromatin packing as a result of the apparent absence of histone $\mathrm{H} 1$ (for reviews see $[17,18])$. Given recent findings that suggest the spatial organization of chromatin within the nucleus affects gene expression [19], it appears likely that dramatic macro-scale rearrangement of chromatin accompanied by micro-scale nucleosome rearrangements over IGR play a major role in directing the cascade of developmentallylinked mRNA steady state levels during intraerythrocytic schizogony. Spatial variations in nucleosome occupancy in $P$. falciparum are generally apparent over key expression landmarks $[16,20]$. Nucleosomes are preferentially distributed over exonic sequences whilst intergenic regions immediately flanking exonic sequences are relatively nucleosome depleted [14-16]. To date, however, no evidence of a spatial correlation between poly dA.dT tracts and the positioning of these gene-flanking NFR has been demonstrated. Moreover, whilst in other organisms the 5 ' flanking NDR typically contain the transcription start site, this is unlikely in P. falciparum. Precise mapping of transcriptional start sites is challenging within the extreme AT nucleotide content of $P$. falciparum intergenic regions (typically exceeding 80-90\%), although a recent study performed by us suggests that these are likely located some 600-1350 bp upstream of the start of the coding sequence [21-23], at least 300-400 bp beyond the mapped 5' NDR border.

The global impact on human and animal health imposed by the protist parasites of the Apicomplexan phylum, of which $P$. falciparum is perhaps the best-known member, has driven a sustained effort to sequence their genomes over recent years. Thus, complete annotated genomes are available for: (i) the human malaria parasites P. falciparum, $P$. vivax and $P$. knowlesi as well as murine models for this disease ( $P$. berghei and $P$. yoelii), (ii) the closely related hematozoans of the order Piroplasmarida Theileria spp. and Babesia bovis, aetiological agents of the bovine diseases tropical theileriosis, East Coast fever and babesiosis, (iii) three genomes for Cryptosporidium spp., aetiological agents of a life-threatening diarroheal disease in immunocompromised individuals and (iv) two additional coccidian parasites of the sub-order Eimeriorina, Toxoplasma gondii and Neurospora caninum, responsible for abortive diseases in sheep and cattle, respectively (see Figure 1 for cladogram of these organisms) [20,24-33]. These resources offer an opportunity to perform a detailed comparative analysis of homopolymeric tract organisation within IGR distinct from previous comparative studies that have emphasised evolutionary diversity in the organisms investigated. In a recent report exploring the size and organisation of IGR within the Apicomplexan phylum, we demonstrated a consensus gene-spacing rule that is shared between the moderately compact genomes in this phylum despite the

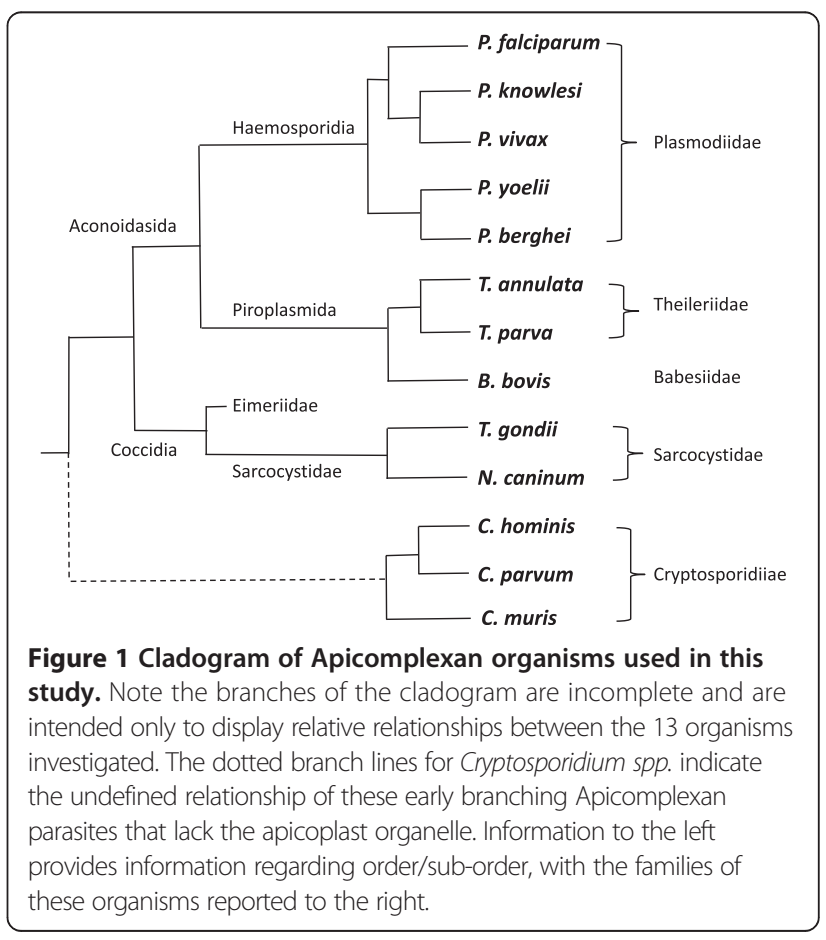


huge variation in the sizes of their genomes (8-63Mbp) [22]. That is, the size of IGR reflects the nature of the core transcriptional activity over the IGR; group A IGRs, containing two promoters (head-to-head flanking genes), are larger than group B IGRs, which contain one promoter and one terminator (head-to-tail flanking genes), and these in turn are larger than type C IGRs that contain two terminators (tail-to-tail flanking genes). For those parasite genomes with moderately compact gene density (c. 2.0$4.8 \mathrm{kbp} / \mathrm{ORF}$ ), we showed that irrespective of the actual mean sizes of the IGR there is a consensus 3:2:1 ratio in the median size of types A:B:C IGR.

This study was designed to address two aims. First, provide a comparative analysis of homopolymer tract frequency, size and spatial organisation in what may be considered functionally comparable regions of flanking IGR in these evolutionary-related pathogenic organisms. The second aim then correlates the spatial organisation of poly dA.dT tracts in $P$. falciparum with available nucleosome mapping data to explore a role for poly $\mathrm{dA}$. $\mathrm{dT}$ tracts in directing nucleosome positioning over NDR associated with key expression landmarks.

\section{Results}

Comparative analysis of the representation of homopolymer tract frequencies in the proximal intergenic regions of

\section{Apicomplexan parasites}

To explore homopolymeric tract organisation in functionally-comparable regions of flanking IGR, the median size of type A IGR (promoter) and type C IGR (terminator) of sense-strand flanking sequences upstream and downstream, respectively, of each ORF in 13 Apicomplexan parasite species were obtained (Additional file 1: Table S1). In each case, flanking sequences were obtained up to a maximum length (correlating to the median size of the IGR as indicated in Additional file 1: Table S1) unless the adjacent ORF was encountered, in which case, only the flanking sequence up to the ORF was taken. The open source algorithm Poly (see Methods) was used to search for and provide quantitative data on the frequencies of homopolymer tracts nucleotides A, G, C and T (collectively termed i) for all lengths $\mathrm{N}$ [3,34]. These data include: (1) the fraction of each nucleotide $\mathrm{i}$ within these sequences $\left(\right.$ Fraction $\left._{\mathrm{i}}\right),(2)$ the frequency of each tract $\mathrm{i}$ of length $\mathrm{N}$ bases observed $\left(f \mathrm{i}_{\mathrm{N}} \mathrm{Obs}\right)$ as well as that expected $\left(f \mathrm{i}_{\mathrm{N}} \mathrm{exp}\right)$ from randomized sequences of the same length and nucleotide content, (3) the maximal length of each homopolymer tract observed $\left(\mathrm{N}_{\max } \mathrm{obs}\right.$, where the occurrence of $\mathrm{N}_{\max } \mathrm{obs}$ was $\geq 4$ ) and that expected $\left(\mathrm{N}_{\max } \exp \right)$, again, from randomized sequences of the same length and nucleotide content (Figure 2 and Table 1). These data enable various aspects of the relative frequency and length of all different homopolymer tracts in these proximal flanking regions to be described. First, their

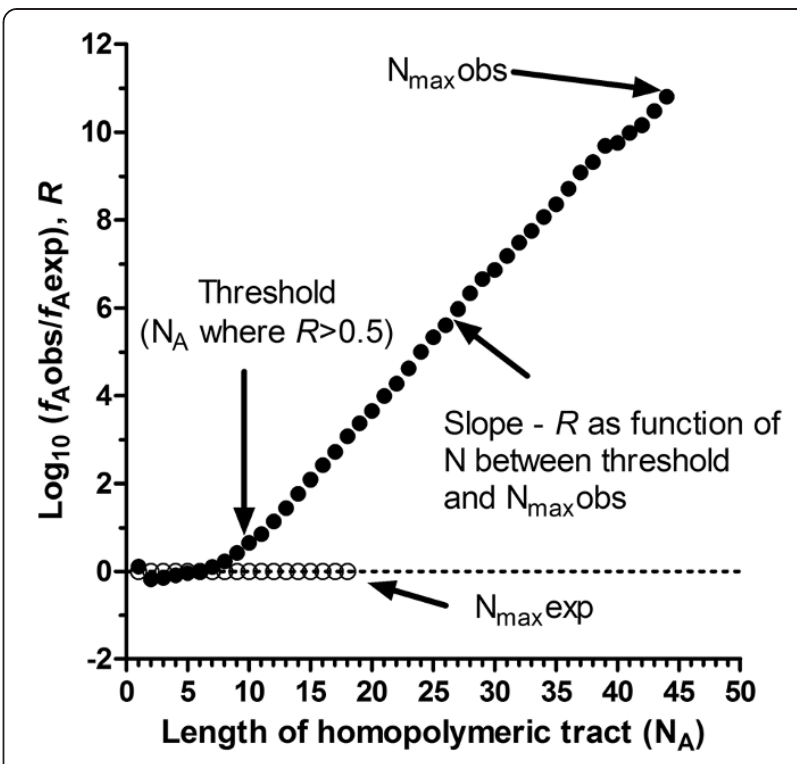

Figure 2 Example output from Poly analysis of homopolymer tract frequency. The representation, $R_{r}\left(\mathrm{f}_{\mathrm{A}} \mathrm{obs} / \mathrm{f}_{\mathrm{A}} \exp \right)$ of poly $\mathrm{dA}$ tracts is plotted as a function of their length $\left(N_{A}\right)$. Filled circles represent the data obtained from an analysis of up to 2000 bases of sense-strand sequence upstream of open reading frames in P. falciparum. Unfilled circles represent data obtained from randomised shuffled sequences of the same length and nucleotide content. $\mathrm{N}_{\max }$ obs is the longest length of poly dA observed (where a minimum of four tracts of this length were observed) in the upstream sequence, with $\mathrm{N}_{\max }$ exp representing the maximal length of poly $\mathrm{dA}$ tract expected from random base sequence of the same length and nucleotide content. The threshold is the length of poly $\mathrm{dA}$ tract which is observed to be over-represented (using the $R>0.5$ criterion) in the upstream sequences. The slope of overrepresentation $\left(\mathrm{slope}_{R}\right)$ is determined from data between the threshold and $\mathrm{N}_{\max } \mathrm{Obs}$ points.

representation, $R$, which provides a measure of the observed frequency of each tract length $\left(f \mathrm{i}_{\mathrm{N}} \mathrm{Obs}\right)$ normalised against that expected by random occurrence ( $\left.f \mathrm{i}_{\mathrm{N}} \exp \right)$. Given that homopolymeric tracts in intergenic regions are highly overrepresented, this is plotted as $\log _{10}\left(f \mathrm{i}_{\mathrm{N}} \mathrm{Obs} /\right.$ $f \mathrm{i}_{\mathrm{N}} \mathrm{Obs}$ ) vs $\mathrm{N}$ (Figure 2). Another measure involving $R$ is the threshold of overrepresentation, a particular value of $R$ and tract length $\mathrm{N}$ where the relationship $f \mathrm{i}_{\mathrm{N}} \mathrm{Obs}>f \mathrm{i}_{\mathrm{N}} \exp$ achieves significance. Here the threshold is set at $R \geq 0.5$, i.e. the observed frequency is $10^{0.5}$ (3.16-fold) higher than that expected by chance from randomized sequences. The second aspect, proportion $(P)$, provides a description of the relative length of the longest observed tract i compared to that expected from randomized sequences, and is derived from $\mathrm{N}_{\max } \mathrm{obs} / \mathrm{N}_{\max } \exp$ (Table 1).

Plotting $R$ as a function of tract length $\mathrm{N}$ reveals three distinct organisations of homopolymer tracts in the proximal intergenic flanking regions of these Apicomplexans, with related organisms generally sharing the same organisation (Additional file 1: Figure S1 and S2). The first, shared by the Plasmodium spp. and Cryptosporidium spp., 
Table 1 Summary of POLY analyses of upstream and downstream gene-flanking sequences in Apicomplexan parasites

\begin{tabular}{|c|c|c|c|c|c|c|c|c|c|c|c|c|c|c|c|c|c|c|c|c|c|c|c|c|}
\hline \multirow[t]{2}{*}{ Organism } & \multicolumn{4}{|c|}{ Fraction $_{\mathrm{i}}$} & \multicolumn{4}{|c|}{$\begin{array}{l}\text { Maximum length } \\
\text { observed } \mathrm{N}_{\max } \text { obs }\end{array}$} & \multicolumn{4}{|c|}{$\begin{array}{l}\text { Maximum length } \\
\text { expected } M_{\max } \text { exp }\end{array}$} & \multicolumn{4}{|c|}{ Proportionment $(P)$} & \multicolumn{4}{|c|}{$\begin{array}{l}\text { Threshold } \\
(R>0.5)\end{array}$} & \multicolumn{4}{|c|}{ Slope $_{R}^{1}$} \\
\hline & A & C & G & $\mathrm{T}$ & $A$ & $\mathrm{C}$ & G & $T$ & A & $\mathrm{C}$ & G & $\bar{T}$ & A & C & G & $T$ & $\bar{A}$ & $\mathrm{C}$ & $G$ & $\bar{T}$ & A & $\mathrm{C}$ & $\bar{G}$ & $T$ \\
\hline \multicolumn{25}{|l|}{ Ipstrear } \\
\hline P.falciparur & 3 & 6 & 7 & ( & & 9 & 9 & 4 & 1 & 5 & 5 & 19 & 4 & 1.80 & 1.80 & 2.26 & 10 & 3 & 4 & 10 & 0.31 & 72 & 74 & \\
\hline .knowlesi & 0 & 19 & 18 & 0.31 & 46 & 17 & 17 & 47 & 1 & 9 & 9 & 13 & 3.54 & 1.89 & 1.89 & 3.62 & 7 & 5 & 5 & 7 & 0.46 & 49 & 0.48 & 0.4 \\
\hline thy & & 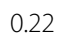 & 0.21 & 0.29 & 35 & 17 & 16 & 3 & 12 & 10 & 10 & 12 & 2 & 1 & 0 & 3 & 6 & 5 & 5 & 6 & 7 & 36 & 5 & 0.45 \\
\hline oeli & 0 & 0.10 & 0.12 & 0.36 & 35 & 10 & 7 & 32 & 18 & 7 & 7 & 6 & 1.94 & 1.43 & 1.00 & 2.00 & 10 & 4 & 5 & 8 & 0.32 & 0.57 & 0.40 & 0.3 \\
\hline Dheraho & 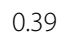 & 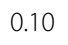 & ) & ( & 2 & 10 & r & 2 & 16 & 6 & . & 16 & 1.44 & 1.67 & 1.17 & 1.50 & 8 & 4 & . & 8 & 25 & 0.57 & 0.48 & 0. \\
\hline (1) & .36 & .14 & 0.15 & 0.35 & 18 & $y$ & $y$ & 18 & 1 & 7 & 1 & 1. & 1.29 & 1.29 & 1.29 & 1.38 & 9 & 5 & 0 & 8 & 0.24 & 0.46 & 0.39 & 0. \\
\hline (at & 0.37 & 0.13 & 0.14 & 0.36 & 22 & 9 & 10 & $n a^{2}$ & 14 & 7 & 7 & na & 1.57 & 1.29 & 1.43 & na & 9 & 5 & 5 & na & 0.26 & 0.45 & 0.47 & 等 \\
\hline 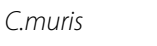 & 0.36 & 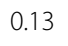 & . & 0.37 & 20 & 10 & 11 & 21 & 14 & 7 & 7 & 14 & 3 & 1.43 & 1.57 & 1.50 & 10 & 6 & 6 & 10 & 0.30 & 0 & 57 & c \\
\hline .gondii & 0.23 & 0.26 & 0.26 & 0.25 & 13 & 17 & 17 & $n$ & 1 & 12 & 12 & na & 1.18 & 1.42 & 1.42 & na & 6 & 10 & 10 & na & 0.08 & 0.29 & 0.29 & a \\
\hline (c) & & 0.27 & 0.26 & 0.25 & 11 & 20 & 21 & 11 & 10 & 12 & 12 & 11 & 0 & 1.67 & 1.75 & 1.00 & J & 10 & 10 & 6 & 0.14 & 0.47 & 0.51 & 0.1 \\
\hline or & 0. & 0.19 & 0.20 & 0.30 & 9 & 7 & 7 & 10 & 12 & 8 & 8 & 12 & 0.75 & 0.88 & 0.88 & 0.83 & na & na & na & na & na & na & na & na \\
\hline Tannul & 0.37 & 0.13 & 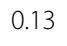 & 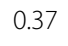 & 11 & 6 & 6 & 11 & 14 & 6 & 7 & 14 & 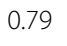 & ) & 6 & 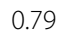 & na & 4 & 7 & na & ב & na & na & \\
\hline arva & & 4 & 15 & 0 & 9 & 6 & 6 & 9 & 1 & 7 & 7 & 13 & & 0.86 & 0.86 & 0.69 & r & 5 & na & na & a & & a & na \\
\hline \multicolumn{25}{|l|}{ Downstream } \\
\hline .raicipe & 0.41 & 7 & 0.07 & 0. & 43 & 7 & 8 & 45 & (1) & 3 & 3 & 18 & 2.53 & 1.40 & 1.60 & 2.50 & 10 & 4 & 4 & 10 & 0.32 & 0.59 & 0.63 & c \\
\hline 列 & 0.29 & 0.19 & 0.19 & 0.3 & 42 & 16 & 16 & 4 & 12 & 9 & 8 & 13 & 3.50 & 1.78 & 2.00 & 3.38 & . & 5 & 5 & 7 & 0.46 & 0.49 & 0.48 & 0.4 \\
\hline s. & & 23 & 0.22 & 0.2 & J2 & 15 & 16 & 列 & 1 & 10 & 10 & 11 & & 1.50 & 1.60 & 2.9 & 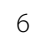 & J & 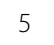 & 6 & 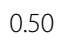 & 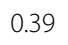 & 40 & \\
\hline yoelii & 0.38 & 0.12 & 0.11 & 0.39 & 33 & 9 & 8 & 31 & 15 & 6 & 6 & 16 & 2.20 & 1.50 & 1.33 & 1.94 & 8 & 4 & 4 & 8 & 0.33 & 0.45 & 0.34 & 0.3 \\
\hline Shorabo & 0.38 & 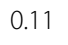 & 0.10 & 0.41 & 2 & 7 & 0 & 2 & 14 & 6 & 6 & 16 & 1.71 & 1.17 & 1.50 & 1.3 & 8 & 4 & 4 & 8 & 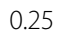 & 4 & 0 & 0. \\
\hline . & 0.35 & 4 & 0.13 & 0 & 13 & 7 & 7 & 10 & 12 & 0 & 6 & 13 & 1.08 & 1.17 & 1.17 & 1.23 & $y$ & 0 & 0 & 9 & 0 & 2 & 9 & 0.2 \\
\hline r noruum & 35 & 13 & 0.13 & 0.39 & 15 & 7 & 7 & 19 & 12 & 6 & 6 & 14 & 1.25 & 1.17 & 1.17 & na & 9 & 6 & 5 & 9 & 0.21 & 0.37 & 0.36 & 0.2 \\
\hline 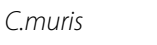 & 0.37 & 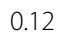 & 2 & 0 & 1 & 0 & 7 & 18 & 15 & 0 & 6 & 14 & 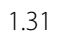 & 1.00 & 1.17 & 1.29 & 10 & 5 & 0 & 11 & 0.30 & 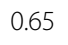 & 0 & 0. \\
\hline . & 0.25 & 0.25 & 0.25 & 0 & 11 & 15 & 17 & 1 & 11 & 11 & 11 & 2 & 1.00 & 1.36 & 1.55 & na & 7 & 10 & 10 & 7 & 0.06 & 3 & 47 & ( \\
\hline - & 0.24 & 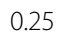 & 0 & 0 & 11 & 21 & 24 & 11 & 11 & 11 & 12 & 11 & 1.00 & 1.91 & 2.00 & 1.00 & 6 & 10 & 10 & 6 & 0.11 & 54 & 51 & 0 \\
\hline bovis & 0.30 & 0.19 & 0.19 & 0.31 & 9 & 7 & 7 & 8 & 11 & 8 & 8 & 11 & 0.82 & 0.88 & 0.88 & 0.73 & na & na & na & na & na & na & na & Ind \\
\hline . & 0.36 & 12 & 0.13 & 0. & 10 & 7 & 6 & 9 & 1 & 6 & 6 & 3 & 0.77 & 1.17 & 1.00 & 0.69 & าa & 5 & na & a & a & $a$ & a & na \\
\hline oarva & 0.35 & 0.13 & 0.14 & 0.38 & 9 & 7 & 6 & 9 & 12 & 6 & 6 & 13 & 0.75 & 1.17 & 1.00 & 0.69 & na & 5 & na & na & na & na & na & Id \\
\hline
\end{tabular}

${ }^{1}$ slope of $R$ between threshold of overrepresentation and $\mathrm{N}_{\max }$ obs. ${ }^{2}$ na, Not available.

shows the typical eukaryotic organisation of overrepresentation of short poly dG.dC tracts and long poly $\mathrm{dA}$. $\mathrm{dT}$ tracts [3]. This pattern is reversed in the second organisation shared by the coccidian parasites $N$. caninum and T. gondii, where instead short poly dA.dT tracts and long poly dG.dC tracts are overrepresented. The final organisation is shared by the piroplasmida Theileria spp. and B. bovis, where there is no evidence of overrepresentation of any homopolymer tracts, with the longest poly dA.dT tracts in Theileria spp. actually reaching the threshold for underrepresentation $(R \geq-0.5)$. Of note, however, is that despite the dissimilarities in the length of proximal upstream and downstream flanking sequence secured from each organism, there appears to be no difference between them in terms of the maximum level of $R$, threshold of overrepresentation or $\mathrm{N}_{\max }$ obs (Table 1 ).

To compare the relative levels of overrepresentation of the different homopolymeric tracts between these species against the backdrop of their diverse nucleotide content, the slope of $R\left(\right.$ slope $_{R}$ ) was determined (Figure 2 and Table 1). In general, as Fraction increases, slope $_{R}$ would decrease as the denominator $f \mathrm{i}_{\mathrm{N}} \exp$ increases. Comparison of slope $e_{R}$ for all homopolymeric tract types in upstream and downstream intergenic flanking sequences (Figure 3A) reveals that this general trend is present $\left(r^{2}=0.33, p<0.001\right.$, slope $\left.=-0.80\right)$. However, reanalysis of this trend for each homopolymer type reveals no significant correlation, excepting polydC in the upstream region (Figure 3A). Thus, the three distinct organisations of 

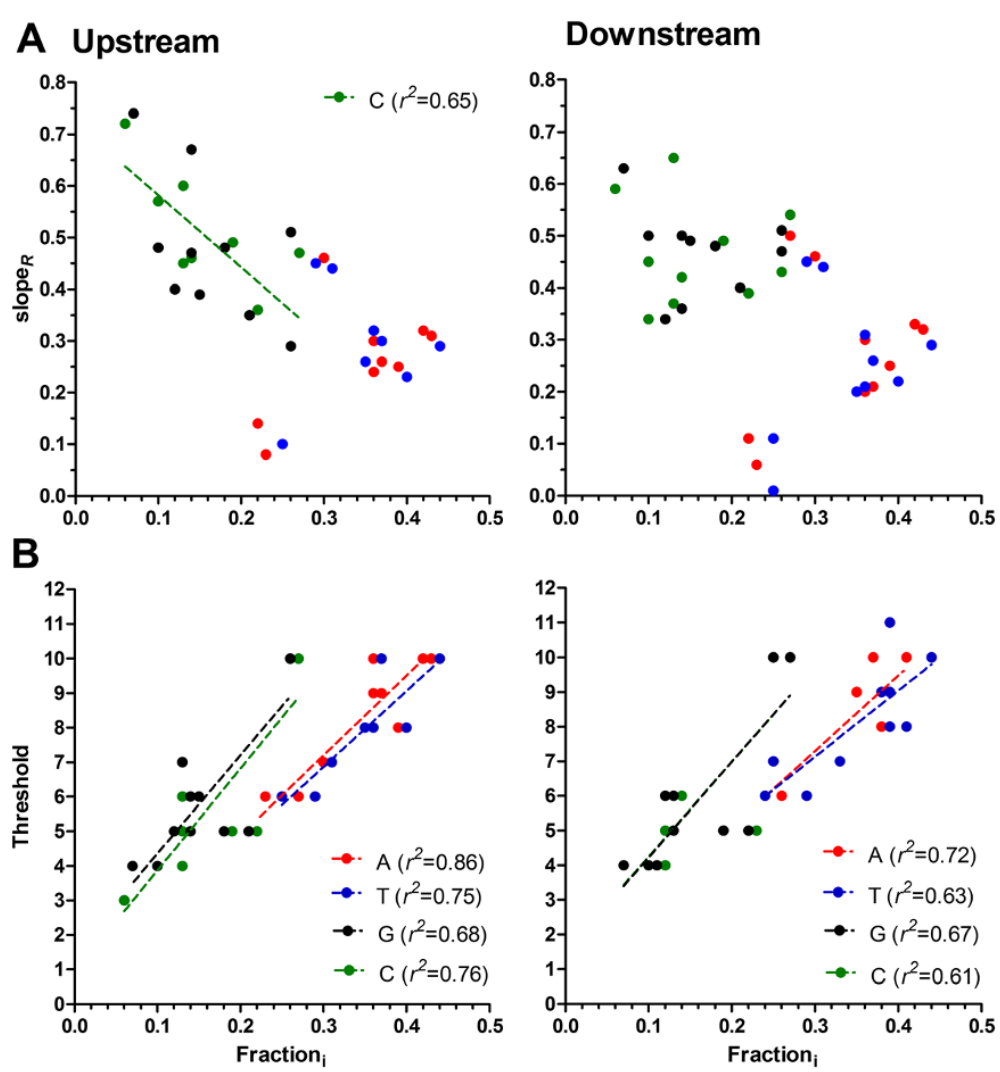

Figure 3 Comparative analysis of overrepresentation of homopolymeric tracts as a function of nucleotide content. (A) The slope of overrepresentation (slope ${ }_{R}$ ) of each homopolymer tract (see key in B) plotted as a function of the fraction of each nucleotide content (Fraction ${ }_{i}$ ) in upstream and downstream proximal flanking regions. Only poly dC tracts in upstream proximal flanking regions show a significant correlation as indicated. (B) The threshold of homopolymer tracti plotted as a function of Fraction in upstream and downstream proximal flanking regions. All linear regression analyses (dotted lines) were significant, with the coefficient of correlation $\left(r^{2}\right)$ reported in the key.

overrepresented homopolymeric tracts is not a reflection of any difference in nucleotide content between these organisms, but instead appears to reflect an inherent difference in the way homopolymer tracts are organised within the different families of the Apicomplexa phylum.

Previous analysis of the threshold length for overrepresentation of homopolymeric tracts in eukaryotic intergenic sequences reveals a positive correlation with Fraction $_{\mathrm{i}}$ $[1,3]$. That is, as Fraction $_{i}$ increases the length of $\mathrm{N}_{\mathrm{i}}$ necessary to meet the threshold of overrepresentation also increases. This is also apparent here (Figure 3B, all regression lines $\mathrm{p}<0.01$ ) in the more defined proximal intergenic sequences investigated in these Apicomplexan organisms, irrespective of any differences in how the homopolymer tracts are overrepresented (nb. Piroplasmida are not included in this analysis as homopolymeric tracts are not overrepresented). Regression analysis reveals no significant difference between the slopes of poly dA.dT or poly dG.dC when compared between upstream and downstream proximal intergenic sequences (all $p>0.15$ ). The slopes estimated for poly dA.dT and poly dG.dC tracts are, however, significantly different from each other $(p<0.0001)$, which would suggest that at an equivalent Fraction $_{\mathrm{i}}$, poly dA.dT are more likely overrepresented at a shorter tract threshold than are poly dG.dC tracts.

\section{Overproportionment of poly dA.dT tracts correlates with the size of flanking intergenic regions in more compact genomes}

The value of $\mathrm{N}_{\max } \mathrm{Obs}$ for any tract type would be expected to increase as both Fraction $_{i}$ and the length of the sequences being investigated increase. Using the denominator $\mathrm{N}_{\max } \exp$ to define proportion, $P$, $\left(\mathrm{N}_{\max } \mathrm{obs} / \mathrm{N}_{\max } \exp \right)$ facilitates a comparative analysis of maximum homopolymer tract length, independent of nucleotide composition and tract length. Plotting $\mathrm{N}_{\max }$ obs of poly dG.dC tracts as a function of either Fraction ${ }_{i}$ or the median size of intergenic sequence (nb. as this increases, longer sequence lengths are obtained for analysis) reveals the anticipated positive correlation in both upstream and downstream proximal flanking sequence populations (Figures $4 \mathrm{~A}$ and $5 \mathrm{~A}$ ). Normalising $\mathrm{N}_{\max }$ obs to determine $P$, reveals the expected levels of overproportionment of poly dG.dC tracts ( $P$ between 0.8 to 1.9 ) in intergenic regions (Figure $4 \mathrm{~B}$ ). $P$ for poly dG.dC 

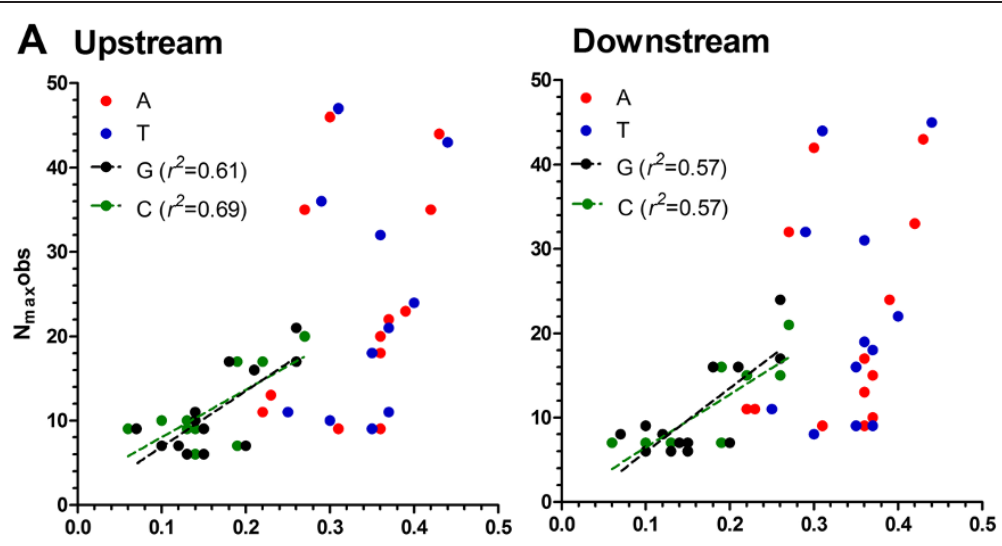

B
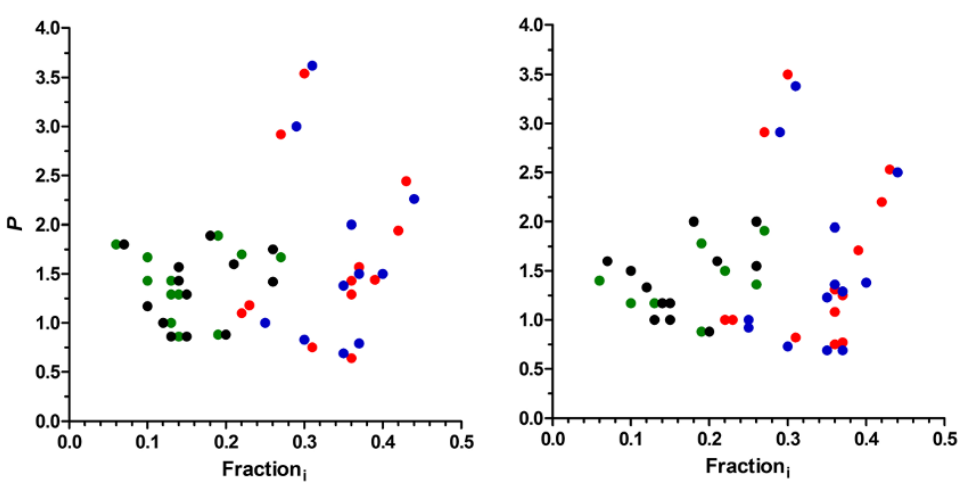

Figure 4 Comparative analysis of overproportionment of homopolymeric tracts as a function of nucleotide content. Plots of (A) $N_{\max }$ obs and (B) proportionment (P) of each homopolymer tract (see key in A) as a function of Fraction, in upstream and downstream proximal flanking regions. Only the $\mathrm{N}_{\max }$ obs of poly dG.dC tracts show a significant linear correlation. This is represented using dotted lines with the coefficient of correlation $\left(r^{2}\right)$ reported in the key.

tracts does not correlate with nucleotide composition, and whilst there is a significant coefficient of correlation between $P$ and length of intergenic regions, the negligible elevation of the slope suggests $P$ is largely independent of length of the upstream and downstream proximal intergenic sequences investigated here (Figure 5B).

The $\mathrm{N}_{\max }$ obs of poly dA.dT tracts as a function of

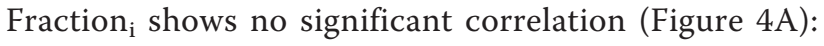
i.e. the maximum length of poly dA.dT tracts is not simply dependent upon how AT rich a genome is. The highly overproportioned ( $P$ between 1.44 and 3.62) poly dA.dT tracts found in Plasmodium spp., irrespective of the AT-content in the proximal flanking regions, clearly biases this analysis (Figure 4B). Plotting $\mathrm{N}_{\max }$ obs as a function of the median intergenic distance also initially reveals no significant correlation, primarily due to the absence of overproportioned poly dA.dT tracts in the large intergenic distances found in T. gondii and N. caninum. Here, the proportionally longer type $C$ intergenic regions (two terminators) in $T$. gondii and $N$. caninum collapses the 3:2:1 space apportionment ratio typical of the other Apicomplexan parasites investigated (Additional file 1: Table S1), all of which share a more compact genome density (2.0-4.6 kb/gene) than that of these coccidian parasites (8.6-9.1 kb/gene) [22]. Excluding $T$. gondii and $N$. caninum, however, reveals a strong positive correlation between both $\mathrm{N}_{\max }$ obs and $P$ of poly dA.dT tracts as intergenic distances increase in length (Figure 5, fit lines shown). Thus, the overproportionment of the long poly dA.dT tracts in Plasmodium spp. is not a reflection of any bias in their AT content, but is perhaps instead a reflection of the longer lengths of their intergenic sequences. This observation goes some way to explain why the $\mathrm{N}_{\max } \mathrm{Obs}$ of poly dA.dT tracts in $P$. falciparum and $P$. knowlesi are similar; despite $P$. knowlesi proximal flanking sequences having a much lower AT-content than those of $P$. falciparum, as the median intergenic distances in $P$. knowlesi are longer.

\section{Homopolymer tracts are overrepresented and overproportioned in the open reading frames of Plasmodium spp}

Previous analysis of homopolymer tract organisation in $P$. falciparum ORF reports the overrepresentation and overproportionment of all homopolymer tract types, but poly dA.dT tracts in particular $[1,3]$. To extend this 


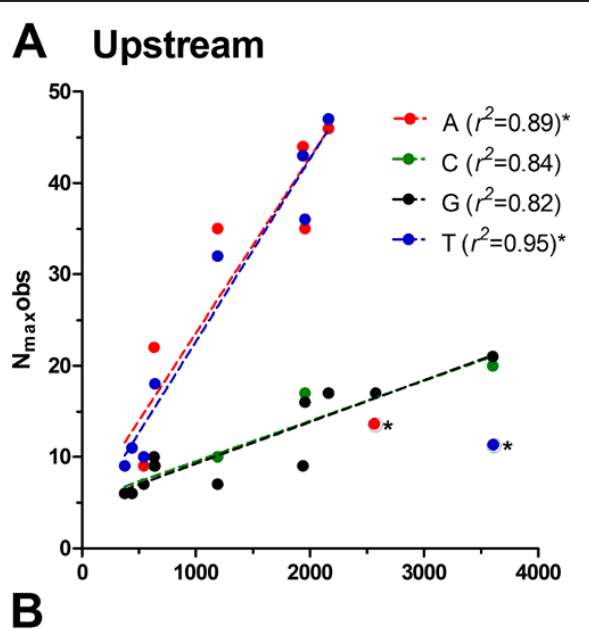

\section{Downstream}
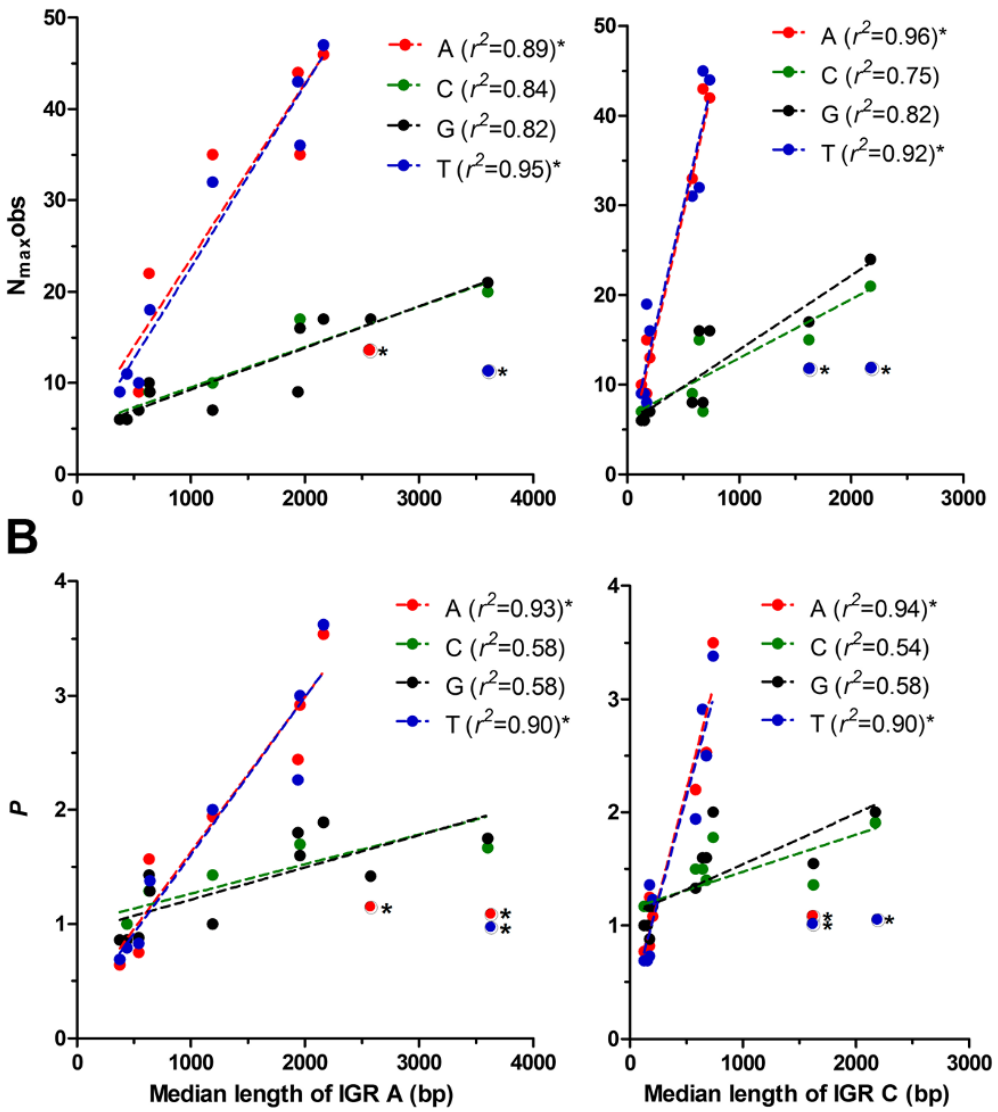

Figure 5 Comparative analysis of overproportionment of homopolymeric tracts as a function of size of intergenic region (IGR). Plots of (A) $\mathrm{N}_{\max } \mathrm{Obs}$ and (B) proportionment (P) of each homopolymer tract (see keys) as a function of the median length of upstream (type A IGR) and downstream (type C IGR) proximal flanking regions. All significant linear correlations are represented using dotted lines with the coefficient of correlation $\left(r^{2}\right)$ reported in the respective key for that panel. Note, poly dA.dT points indicated with asterisks (T. gondii and N. caninum) were omitted from the linear regression analysis; the $r^{2}$ reported also omits these values).

analysis, ORF were obtained for all (except $N$. caninum) of the Apicomplexan parasites investigated here and the program Poly was used to search for and provide quantitative data for the frequency of all homopolymer tract types (Additional file 1: Table S1). For comparison, a Poly analysis of human and mouse ORF were included, as these organisms represent the general eukaryotic pattern of absence of any homopolymeric tracts in these sequences $[1,3]$.

Our analysis reveals that, like human and mouse ORF, the ORF of cryptosporidium, piroplasmida and coccidian parasites show no evidence of overrepresented or overproportioned homopolymeric tracts (Figure 6 and Additional file 1: Figure S3). In fact, these tracts instead tend to be underrepresented and underproportioned, particularly at longer tract-length, reflecting the impact of codon usage in these coding sequences. All the Plasmodium spp., however, show extensive overrepresentation and overproportionment of all homopolymeric tracts in
ORF sequences, and again of poly dA.dT in particular (Additional file 1: Figure S3). As expected, overrepresentation and overproportionment of tracts is greater in human malarial parasites (eg. poly dA.dT $\mathrm{N}_{\max }$ obs range between 29-42 with $P$ between 2.1-3.82) than murine malarial parasites (eg. poly dA.dT $\mathrm{N}_{\max }$ obs range between 17-29 with $P$ between 1.31-1.69) reflecting the larger average size of ORF in human compared to murine malarial parasites.

\section{Spatial analysis of poly dA.dT tracts in proximal flanking intergenic sequences}

Given the distinct overrepresentation and overproportionment of poly dA.dT tracts in the proximal intergenic sequences of three of the six Plasmodium spp. that are the aetiological agents of human malaria, these organisms were selected for an initial analysis of the spatial organisation of these homopolymer tracts using the frequency counting program motif.freq.pl (see Methods). Upstream 

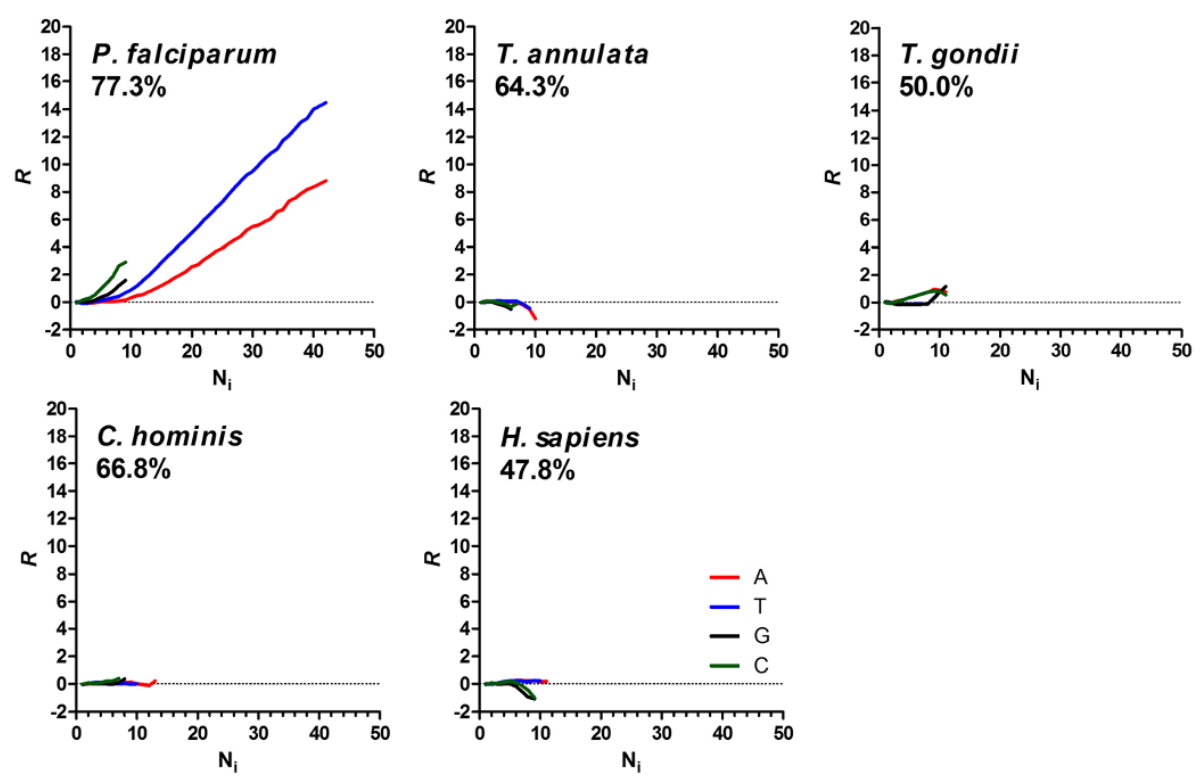

Figure 6 Representation of homopolymeric tracts over coding sequences. These graphs plot $R$ for homopolymer tracts (see key, lower right) as a function of their length $\left(\mathrm{N}_{\mathrm{i}}\right)$. The species as well as the average\%AT content of the coding sequences analysed is reported on each graph.

(2000 bases) and downstream (700 bases) sense-strand sequences for all genes were distributed into sequential 50 base length bins for a count of the occurrence of non-overlapping poly dA.dT tracts of $\mathrm{N}=5,10,15$ and 20 base length. Given that the total number of nucleotides in each bin will decrease as the distance of the bin from the ORF increases ( $\mathrm{nb}$. the probability of encountering an adjacent ORF increases as you move away from the ORF of interest, and only intergenic sequence are captured for analysis here), the number of tracts observed is normalised by the total number of nucleotides in the bin (representing the bin frequency counting output of motif.freq.pl) to provide a useful comparative measure of the observed frequency $\left(\mathrm{F}_{\mathrm{obs}}\right)$ of these non-overlapping tracts.

Comparison of the $\mathrm{F}_{\mathrm{obs}}$ of the different lengths of poly dA.dT tracts in both upstream and downstream proximal flanking regions shows the expected decrease as (i) the Fraction $_{\mathrm{i}}$ of nucleotides A and $\mathrm{T}$ decreases from P. falciparum to P. vivax and (ii) as the length of tract investigated increases (Figure 7). Irrespective of the Plasmodium spp. investigated, the spatial distribution in $F_{o b s}$ for all tract lengths only varies in the flanking regions immediately adjacent to the ORF (i.e. within $200 \mathrm{bp})$. A higher-resoultion determination of $F_{\text {obs }}$ was therefore carried out on the 200 bases that flank either side the start and end of all ORF in these Plasmodium $s p p$. to refine this observation (Figure 8 , tract size $\mathrm{N}=5$, 10 base bins). This analysis reveals a clear pattern of spatial arrangement of poly dA.dT tracts on the sense strands immediately prior to and following the ORF. Poly dA tracts are more likely observed within 10-20 bp either side of the ORF, with peaks of $F_{\text {obs }}$ for poly $\mathrm{dT}$ tracts more distally located, approximately $50 \mathrm{bp}$ upstream of the start codon and 50-200 bp downstream of the stop codon. Accounting for the differences in AT content between these three Plasmodium spp., an "expected" frequency of occurrence of $\mathrm{N}=5$ poly dA.dT tracts can be estimated from a repeated $10 \times$ random shuffle of the sequences using the program shuffle.pl (see Methods), where the 10X shuffled frequencies are averaged to produce $F_{10 x s h u f f l e}$ ensuring that the Fraction ${ }_{\mathrm{i}}$ of all nucleotides is maintained during shuffling but not the integrity of homopolymer tracts. Plotting the normalized frequency ratio, $F_{\text {obs }} / F_{10 x s h u f f l e}$, accounts for base composition bias and the plots show that as the Fraction $_{\mathrm{i}}$ of $\mathrm{A}$ and $\mathrm{T}$ nucleotides decreases from $P$. falciparum to $P$. vivax, the ratio for these peaks actually becomes greater (Additional file 1: Figure S4). That is, these spatial features of poly dA.dT tract organisation are inherent structural features and occur irrespective of AT-bias.

Genome-wide maps of nucleosomal occupancy are available for P. falciparum [14,15]. To compare nucleosome occupancy with these spatially organised poly dA. $\mathrm{dT}$ tracts around the translational start and stop sites, a $\log _{2}$ ratio of existing next generation high throughput sequence reads from formaldehyde-assisted isolation of regulatory elements, FAIRE, (representing nucleosome free DNA) and micrococcal nuclease-assisted isolation of nucleosomal elements, MAINE, (representing nucleosome bound DNA) are provided to indicate relative nucleosome deficiency over this region [20]. As expected from similar analyses in other eukaryotes, the position of the proximal flanking poly dA.dT tracts correlate with the borders of 


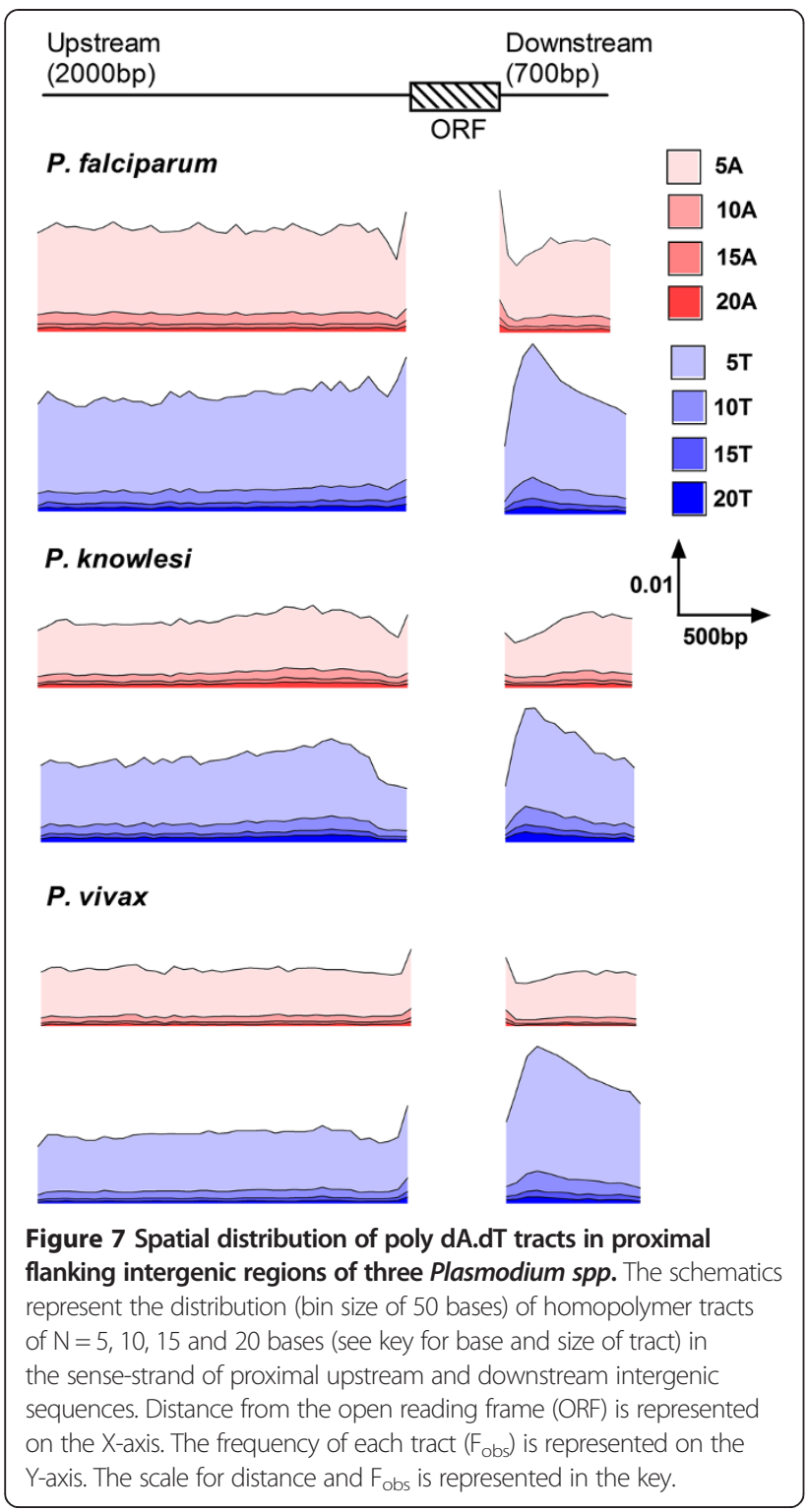

the NDR located both upstream and downstream of the ORF (Figure 9).

Interestingly, this same relative spatial organisation of poly dA.dT tracts immediately adjacent to the ORF is found in the other Apicomplexan species (Additional file 1: Figure S5) investigated here. Thus, whilst baseline $F_{o b s}$ values may vary, reflecting known relative differences in overrepresentation of poly dA.dT tracts, there appears to be an established spatial organisation of poly dA.dT tracts immediately adjacent the start and end of an ORF in Apicomplexan parasites.

\section{Spatial analysis of poly dA.dT tracts over the core promoter in $P$. falciparum}

In the absence of unambiguously mapped transcription start sites for $P$. falciparum, available in silico predictions of core promoters were used to explore the spatial arrangement of poly dA.dT at this additional regulatory landmark [35]. Previous nucleosome mapping has revealed a relative deficit of nucleosome assembly centred $50 \mathrm{bp}$ upstream of the most highly predicted core promoters [20]. Taking the same 3477 most confidently predicted $($ EGASP $=1)$ core promoters used to map this nucleosome depleted region, $400 \mathrm{bp}$ of sequence centred on the highest scoring position for the transcription start site were secured here for an analysis of the spatial distribution of poly dA.dT tracts. Using $\mathrm{N}=5$ and $\mathrm{N}=10$-mers (10 base and 25 base bins, respectively) the $\mathrm{F}_{\text {obs }}$ for these poly dA.dT tracts were plotted against the distance from the predicted core promoter (Figure 10). For comparison, a $\log _{2}$ ratio of FAIRE/MAINE reads was overlaid to indicate the relative nucleosome deficiency over this same region. This analysis reveals a peak of poly dT located on the sense-strand some 10-20 bp upstream of the highest scoring position for the transcription start site, coincident with the peak of relative nucleosome deficiency. A second, but less abundant, peak of poly $\mathrm{dA}$ is located on the same strand some 30-50 bp further upstream of the poly dT peak. Extending this analysis to core promoters with more moderate thresholds of confidence (EGASP of 0.4-0.9) retains the same relative spatial organisation of poly dA.dT peaks to the core promoter and position of the nucleosome free region, albeit with lower $\mathrm{F}_{\mathrm{obs}}$ (Additional file 1: Figure S6).

\section{Discussion}

Simple sequence repeats, such as homopolymeric tracts, are important molecular tools in the investigation of genetic disease and evolution. As such, significant efforts have been invested in understanding the forces that shape their frequency, length, spatial distribution and function [1,3,35-37]. It is apparent that there is a dynamic balance between stochastic seeding and expansion of homopolymer tracts, their mutation, and non-stochastic selective pressures (e.g. intrinsic DNA factors such as triplet code, nucleosome positioning information and initiation of transcription), that together direct their evolution and persistence. Two leading theories for the seeding and expansion of homopolymer tracts invoke either slipped-strand replication errors or the action of transposable elements such as retrotransposons [38-41]. The evidence for slipped-strand replication errors directing tract expansion is initially conceptually compelling. In general, representation of homopolymeric tracts increases logarithmically when tract lengths are equal to, or exceed, approximately 7 bases - this figure apparently representing a minimal thermodynamic threshold for slipped-strand errors during replication [1]. This theory, however, was challenged in a study of homopolymeric tract frequencies in 27 organisms which showed that there 


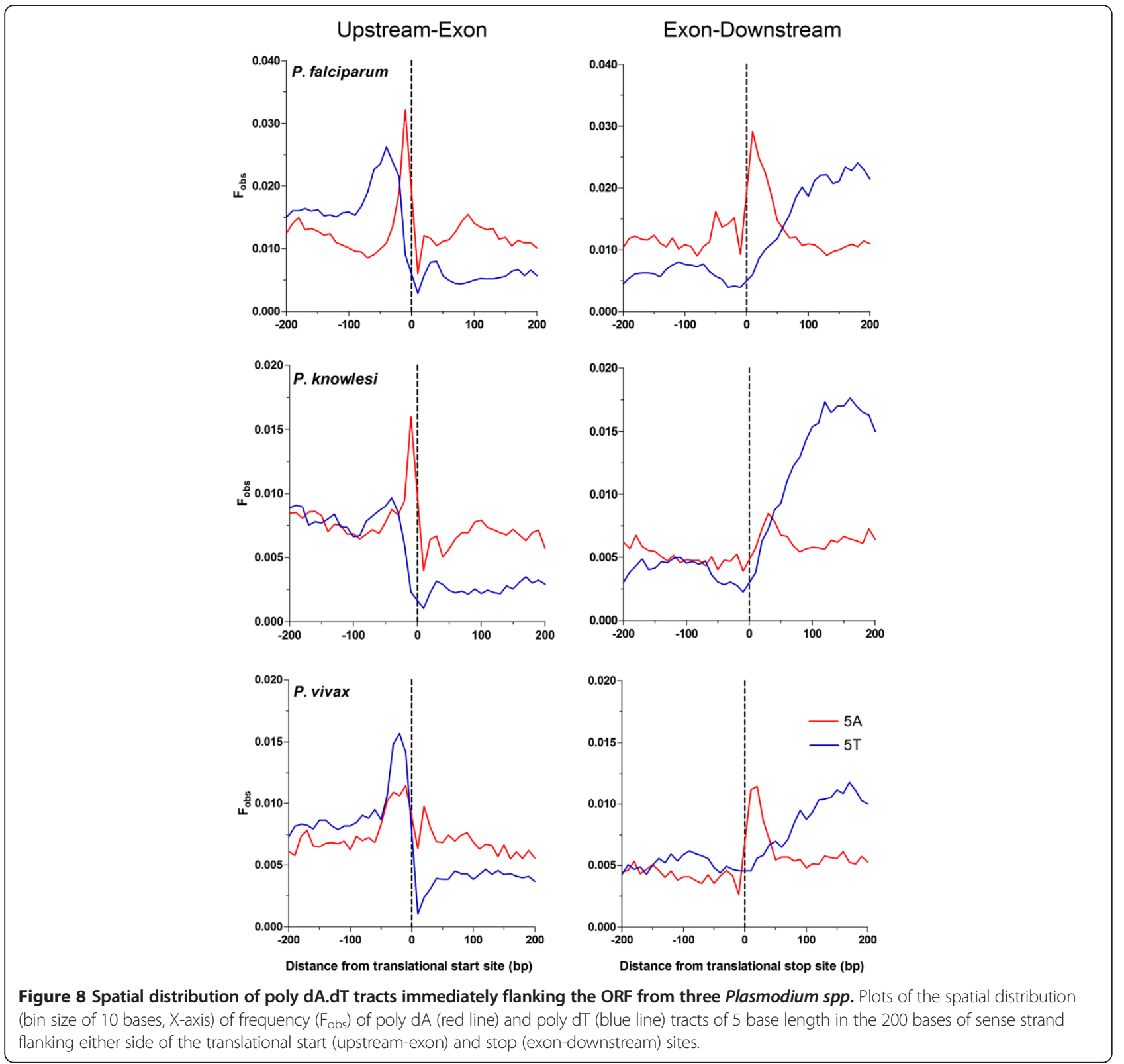

is no single threshold tract-length for homopolymer overrepresentation [3]. Instead, this work demonstrated that the threshold tract-length for overrepresentation is dependent upon the base composition ( Fraction $_{\mathrm{i}}$ ). Thus, for polydA.dT tracts, as Fraction $_{\mathrm{A}, \mathrm{T}}$ increases, the threshold tract length for overrepresentation of these tracts similarly increases. This study of homopolymer tract frequency in Apicomplexan protozoa agrees with this observation, although our study shares with the Zhou et al. study [3] a similar limitation in range of average genomic AT content of organisms (between 50-85\%). Whether this same correlation between threshold tractlength for polydA.dT overrepresentation and Fraction $_{\mathrm{A}, \mathrm{T}}$ exists in more GC rich organisms needs to be established to explore the universality of this observation and its implication for homopolymer tract-length expansion.

The second theory hypothesises that polydA.dT tracts are seeded from polyadenylated transcripts of retrotransposons. This study of Apicomplexan protozoa presents a challenge, although perhaps not insurmountable, to this hypothesis. Whilst transposable elements are ubiquitously found in the genomes of metazoan eukaryotes, and even within lineages of protozoa closely related to Apicomplexans, there is a lack of overwhelming evidence for such transposable elements in the extant genomes of the Apicomplexan organisms investigated here [42-45]. Apicomplexans share the features of relatively small and moderately compact genomes, and are characterised 
A

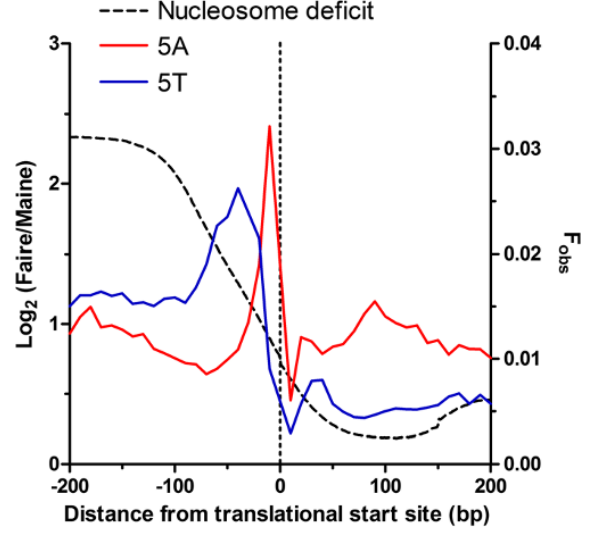

B

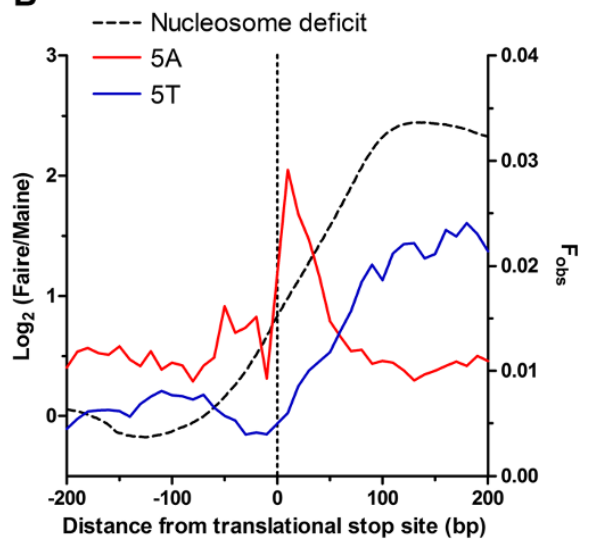

Figure 9 Spatial distribution of nucleosome occupancy and poly dA.dT tracts over sequences immediately flanking $P$. falciparum ORF. Plots of the spatial distribution of observed frequency ( $F_{\text {obs, }}$, right $Y$-axis) of $N=5$ (10 base bins) length poly dA (red line) and poly dT (blue line) tracts over 400 bp centered over translational start (A, upstream-exon) and stop (B, exon-downstream) sites. Nucleosome occupancy (black dotted line) is reported as a $\log _{2}$ ratio of FAIRE to MAINE sequence reads (left Y-axis) where an increase in the ratio indicates regions relatively deficient in nucleosome occupancy.

by gene loss and expansion of species-specific gene families - features attributed to adaptation to a parasitic life cycle and virulence within the host. Critically, whilst there is extensive synteny within families of these parasites (e.g. comparing P. falciparum with P. knowlesi), there is minimal synteny between more distantly related members of the Apicomplexan lineage (e.g. comparing Plasmodium with Cryptosporidium) [42]. This evidence of dramatic genomic rearrangements within ancestral lineages, much more so than in other eukaryotic lineages of similar evolutionary age, coupled with evidence of widespread intron loss during ancestral Apicomplexan genome evolution, have led to the suggestion that retrotransposons were present in Apicomplexan ancestral lineages and may therefore represent the original source of polydA.dT tracts in modern genomes. That said, Plasmodium spp. and Theileria spp. share a recent common ancestor, and show diametrically opposed patterns of homopolymeric tract arrangement in their modern genomes. Thus, any model based on the action of retrotransposons seeding homopolymer tracts within ancestral Apicomplexan lineages would also have to account for the apparent absence of overrepresented homopolymeric tracts in modern Theileria spp. genomes.

Previous comparative studies of homopolymer tract frequency and/or positioning have emphasized phylogenetic diversity in the organisms studied, quite distinct to this study limited to a single phylum [1,3]. However, this study reports features of homopolymer tract frequency, length and distribution common to these previous comparative analyses. Specifically, the correlation between base composition and length of sequence investigated with homopolymer tract representation, thresholds of overrepresentation and degree of overproportionment. Critically, however, analysis of representation and proportion of polydA.dT and polydG.dC tracts within the IGR reveals intriguing, and novel, lineage-specific patterns within Apicomplexans. Whilst members of the Plasmodium and Cryptosporidium families show a greater level of overrepresentation and overproportionment in polyd.A.dT tracts compared to polydG.dC tracts, a pattern generally shared with other eukaryotes, we find a reversal of this pattern in the coccidians and complete absence in the piroplasmida. The apparent deficit of homopolymeric tracts in the piroplasmida cannot be simply accounted for by the relatively short length of the IGR investigated in these organisms (150-550 bp), as short IGR (200-650 bp) are also analysed here in Cryptosporidium spp. [22]. Further, whilst the coccidians have the most GC-rich genomes of the Apicomplexans investigated here, and may therefore be expected to have longer polydG.dC tracts, both humans and mice share a similar base composition and clearly show higher levels of overrepresented and overproportioned polydA.dT tracts in their IGR [3]. Resolution of the impact of these distinct patterns of tract organisation will require nucleosome-positioning data currently not available for these organisms. Of note, however, is that whilst these organisms show distinct patterns of tract organisation in their IGR, the preferential spatial arrangement of polydA.dT tracts immediately flanking ORF is conserved. This organisation presumably confers a comparable role in nucleosome barrier placement at these sites, and suggests that canonical features of an intrinsic nucleosome positioning code are similar in all Apicomplexans.

In many eukaryotes, the 5' NDR contains the transcription start site. Whilst accurate transcription start site mapping data are not currently available for $P$. falciparum, a similar placement of the transcription start site in the 5 ' 

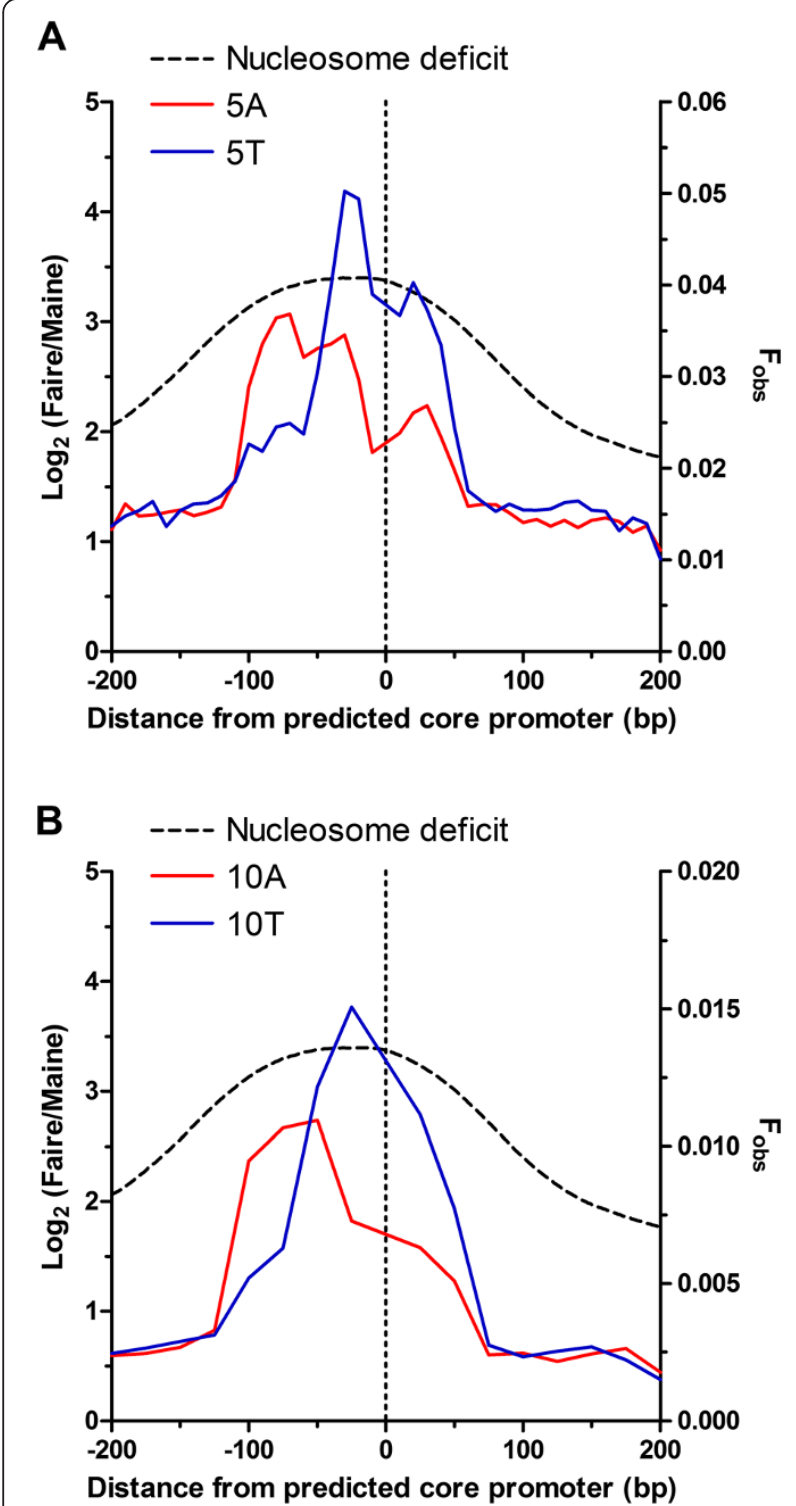

Figure 10 Spatial distribution of nucleosome occupancy and poly dA.dT tracts over predicted core promoters in $P$. falciparum. Plots of the spatial distribution of observed frequency $\left(F_{\text {obs, }}\right.$, right Y-axis) of $\mathrm{N}=5$ (A, 10 base bins) and $\mathrm{N}=10$ (B, 25 base bins) length poly dA (red line) and poly dT (blue line) tracts from a 400 bp region centred over highly predicted core promoters. Nucleosome occupancy (black dotted line) is reported as a $\log _{2}$ ratio of FAIRE to MAINE sequence reads (left $Y$-axis) over the same region where an increase in the ratio indicates regions relatively deficient in nucleosome occupancy.

NDR is unlikely. P. falciparum transcripts contain significant untranslated regions (c. 800-1800 bases), with recent modelling performed by us indicating that some $75-80 \%$ of this is accounted for by the 5'UTR - suggesting that transcription start sites typically lie some 600 to $1350 \mathrm{bp}$ upstream of the translational start site, much further than the 200-300 bp of the 5' NDR [22]. In the absence of mapped transcriptional start sites, we instead correlated nucleosome positional mapping data and the spatial arrangement of polydA.dT with bioinformatic predictions of core promoters $[20,46]$. Some caution needs to be applied to the interpretation of these data, as structural effects of homopolymer tracts on DNA bending are incorporated into the bioinformatic algorithm used to predict core promoters [46]. However, it is noteworthy that the centre of the NDR located over the most confidently predicted core promoters correlates with a peak of polydT on the sense strand, the 3' end of which lies immediately upstream of the likely transcription start site. This arrangement is very similar to that recently described in the similarly AT rich Dictyostelium discodieum [47]. Here, the authors speculate that the RNA polymerase II preinitiation complex exploits the relative instability of the polyrU.dT RNA:DNA hybrid in abortive transcription, a mechanism key for directing a programme of coordinated temporal gene expression. It is therefore noteworthy that a not dissimilar programme of temporal global upregulation of RNAPolII pre-initiation complex (PIC) processivity is found in P. falciparum [48]. During intraerythrocytic schizogony, RNAPolII PIC is constitutively associated with promoter regions, and presumably undergoes repeated rounds of abortive transcription prior to an onset of increased RNAPolII processivity in the early trophozoite stage, some $18-22 \mathrm{~h}$ after the invasion of the host erythrocyte [49]. The molecular basis of this increase in RNAPolII processivity remains to be determined, although conserved features of the T-regulatory loop and/or the heptad repeats within the carboxy-terminal domain of the P. falciparum RNAPolII large subunit would suggest regulation through kinase phosphorylation activity at these sites [50].

Placement of poly dA.dT tracts in the centre of NDR organised over $P$. falciparum promoters supports their role as intrinsic factors in determining nucleosome positioning in these regions. In budding yeast, poly dA.dT tracts have also been demonstrated to act as extrinsic factors in this same role by modulating interactions of promoter DNA with transcription factors, the RNAPolII complex and chromatin remodelling machinery to provide additional context, and directionality, for distinct promoter classes. The data presented here represent the average nucleosome positioning data, and whilst sufficient to demonstrate the intrinsic role, lacks context to explore any extrinsic role. That such a role may exist in P. falciparum is suggested by observed temporal variation in nucleosome positioning over promoters and the preferential positioning of non-canonical histone containing nucleosomes over IGR $[14,15,51]$. Resolution would require additional data correlating temporal gene expression patterns with binding of components of the transcriptional apparatus and/or chromatin remodelling machinery.

A key finding of this study was the highly overrepresented and overproportioned polydA.dT tracts in the 
IGR of the Plasmodium spp. compared to the other Apicomplexans investigated. Significantly, analysis of homopolymer tract organisation over the open reading frames in Plasmodium spp. reveals that polydA.dT are similarly highly overrepresented and overproportioned in these regions. This organisation of polydA.dT over open reading frames is quite distinct from the other Apicomplexans investigated here, and eukaryotic genomes in general $[1,3]$. The presence of polyA tracts within $P$. falciparum open reading frames is well documented; leading to a pronounced codon usage bias and inclusion of low complexity amino acid stretches (particularly polylysine and polyasparagine) within the protein products. This same bias in codon usage is also evident throughout Plasmodium spp., similarly trending towards the inclusion of polyA tracts, although not to the extent of the extremely AT-rich P. falciparum. These low complexity regions (LCR) have been implicated in a variety of regulatory mechanisms including control of translational efficiency, a "smokescreen" of immunodominant regions assisting in the evasion of the host adaptive immune response, or as a protective response to heat-shock [52-56]. Reconciling these diverse adaptive and mechanistic roles for low complexity regions has recently been facilitated by their classification into three distinct groups based on their AT-content and heterozygosity [56]. Poly dA.dT would form part of the polyN group, exhibiting both high AT content and high levels of heterozygosity. No clear role for the polyN class of LCR exists, although speculation for a role in translational efficiency or an equally plausible neutral role has been made. For the neutral role, some form of selection pressure would be required to promote and/or retain these polyN LCR. This pressure may exist if we consider a role for poly dA.dT tracts in the formation of origins of replication in Plasmodium spp., as have been implicated in both budding and fission yeast $[4,6]$, during the critical process of infection and colonisation of the female mosquito following a blood meal on an infected vertebrate host. Plasmodium spp. are haploid for the majority of their life-cycle, with diploid stages only present in early stages of development in the mosquito midgut. Gametogenesis starts in erythrocytes and is completed when the macro- (female) and micro- (male) gametocytes are taken up during a mosquito blood-meal. The final maturation step for the microgametocyte, termed exflagellation, is a remarkable process taking some 20 minutes during which the microgametocyte undergoes three rounds of replication to generate eight flagellated microgametes [57-59]. This rapid replicative cycle would require each of the three rounds of DNA replication to take place over a 3-5 minute window, which, assuming a processivity of the order of $1-2 \mathrm{kbp} / \mathrm{min}$ for eukaryotic replication [60], would require an exceptionally high density of origins of replication throughout the genome. As some
$50 \%$ of Plasmodium spp. genomes encode protein, with a gene density of between 2.6-4.6 kb/gene, these origins of replication likely cannot be restricted solely to IGR, and may therefore provide a novel explanation to account for the significant overrepresentation and overproportionment of polydA.dT observed in both ORF and IGR of Plasmodium spp. genomes.

\section{Conclusion}

Unlike previous comparative studies that emphasise evolutionary diversity in their determination of features of homopolymeric tract representation, length and spatial organisation, this study is instead restricted to a single phylum of unicellular parasites that all share moderately compact genomes. We describe features of polydA.dT tract organisation within this phylum that support a canonical role as intrinsic regulators of nucleosome positioning as well as findings that support nucleotide fraction as a key determinant in the thermodynamic threshold for tract expansion. Critically, we also present evidence for a novel lineage-specific organisation of homopolymer tract organisation in both intergenic and genic compartments along with evidence that overproportionment of homopolymer tract length may be dependent on the available intergenic space into which expand. Given the general lack of specific transcription factors in Apicomplexan genomes, a dynamic programme of nucleosome binding and rearrangement likely plays a significant role in the temporal and absolute regulation of transcription. Our observations relating to polydA.dT spatial arrangement and enrichment likely reflects the impact of chromatin structure and function in shaping the genomes of these parasites important for human and animal health.

\section{Methods}

\section{Homopolymer tract frequency analysis using poly}

Annotated genome sequences for Plasmodium spp. (PlasmoDB.org release 5.5), Theileria spp. and B. bovis (GeneDB.org), Cryptosporidium spp. (CryptoDB.org release 5.5) and $T$. gondii and $N$. caninum (ToxoDB.org release 5.1) were secured from their respective database repositories. Genbank-formatted documents were created that secured specified sizes of intergenic sequences that flank the coding sequence in each genome, taking up to the length specified unless a flanking coding sequence was encountered - in which case only intergenic sequence was secured in a truncated file. These sequence files were then concatenated into ASCII text files, one each for upstream and downstream flanking sequences. The ends of each individual flanking sequence were tagged to prevent the joining of sequences that may create a homopolymeric tract artefact between two sequences. Individual files were analysed using the program Poly [34], open source 
software publically available from www.bioinformatics. org/poly. Non-overlapping homopolymer tracts of all four types are counted by Poly for the entire file and a number of parameters are then calculated, including: the total base count for each file, its GC composition, and the numbers and the frequencies of the homopolymer tracts of different lengths. A moving window of $1 \mathrm{bp}$ in length is used by Poly to differentiate tracts and spacers, taking into account the tags used to prevent artifactual sequence concatenation. These data and additional information are kept as data objects in the program and can be manipulated in various ways. Since Poly has been described in detail in previous reports $[3,34]$, we next only define its parameters that were used in this study.

The observed tract frequency of a given base $\mathrm{i}, f \mathrm{i}_{\mathrm{N}} \mathrm{obs}$, of length $\mathrm{N}$ is determined in Poly by the formula: $f \mathrm{i}_{\mathrm{N}} \mathrm{obs}=$ $c \mathrm{i}_{\mathrm{N}} \mathrm{obs} / l_{\text {seq }}$.

where $c \mathrm{i}_{\mathrm{N}} \mathrm{Obs}$ is the count of observed tracts of base $\mathrm{i}$ at the specific length $N$ contained in each sequence and $l_{\text {seq }}$ is the total length of the sequence (total base count) in which those tracts were counted.

The expected frequency, $f \mathrm{i}_{\mathrm{N}}$ exp, of a homopolymer tract of base $\mathrm{i}$ of length $\mathrm{N}$ randomly occurring is calculated by the formula: $f \mathrm{i}_{\mathrm{N}} \exp =f \mathrm{i}_{\mathrm{N}} \mathrm{obs}^{\mathrm{N}} \times\left(1-f \mathrm{i}_{1} \mathrm{obs}\right)^{2}$.

where $f \mathrm{i}_{1}$ obs is the fractional base composition of that tract base.

The level of tract representation for a given base is then calculated as the ratio of observed to predicted frequencies, defined as Representation, $R: R=f \mathrm{i}_{\mathrm{N}} \mathrm{obs} /$ $f \mathrm{i}_{\mathrm{N}} \exp$.

Tracts are over-represented for $R$ values larger than 1 , and under-represented for values less than 1 . The log $(R)$ vs $\mathrm{N}$ plots were generated using GraphPad Prism v6.0 (GraphPad Software, La Jolla, USA). Linear interpolation of the data was used to determine the values of $\mathrm{N}$ at $\log R$ values of 0.5 (defined here as the threshold for over-representation). The slope of over-representation $\left(\right.$ slope $\left._{R}\right)$ was determined by the same linear interpolation of $R$ between the threshold for over-representation and $\mathrm{N}_{\text {max }} \mathrm{Obs}$ of each tract type. To reduce the noise in the analysis associated with infrequent observations of very long tracts, $\mathrm{N}_{\max } \mathrm{Obs}$ is scored only when $c \mathrm{i}_{\mathrm{N}} \mathrm{Obs} \geq 4$.

The maximum expected length of a homopolymer tract of any base $\mathrm{N}_{\max }$ exp, is calculated for any sequence length based upon random base sequence occurrence in a sequence of that length of equivalent base composition to the real sequence. The maximum homopolymer tract length observed in the real sequence, $\mathrm{N}_{\max }$ obs, can then be compared to its expected length by taking the following ratio, a parameter defined as proportion, $P=\mathrm{N}_{\text {max }}$ obs/ $\mathrm{N}_{\max }$ exp.

The condition of overproportionment is defined for $P$ values larger than 1 , while underproportionment is for $P$ values less than 1 .

\section{Calculating tract frequencies as a function of sequence position}

We developed a non-overlapping motif frequency counting program called motif.freq.pl., written in perl v.5.12.4., which can be accessed at http://www.bioinformatics.org/ motiffreq. It calculates the individual bin frequencies of any specified input sequence motif within a binned sequence. Motif.freq.pl requires the user to enter the input sequence motif to be searched and the bin size. The input sequence(s) is divided evenly into sequence bins of size determined by the input bin size. The program will count the occurrences of the input sequence motif within each bin and return the values to the output file as a motif frequency defined as $\mathrm{F}_{\mathrm{obs}}$, the number of motif occurrences/ nucleotides in the bin. If the motif is repeated in the sequence without interruption by intervening bases, motif. freq.pl will count as many motifs as the program can detect. If the motif is physically located at the bin interface and occurs within two adjacent bins, the motif will be counted as being within the bin which contains the most bases of the motif. If the motif contains an equal number of bases located within two adjacent bins, the motif will be considered to reside within the first bin being counted in the sequence. The output of motif.freq.pl is a text file listing the bin number and motif frequency for each bin.

We also created a sequence-shuffling program that conserves the original input sequence's base frequencies during shuffling. This program is called shuffle.pl, also written in perl v.5.12.4., and it can be accessed at http:// www.bioinformatics.org/motiffreq. The purpose of this program was to assess, by comparison, the significance of the motif.freq.pl program's frequency-bin output resulting from the input of real sequences. The shuffling program reads multiple sequences of specified length in FASTA format and then merges all sequences together into one master combined string. The program then shuffles all bases in the combined string, using the shuffle function in the LIST::Util module in PERL. The shuffled master string is then broken up into the original number and length of sequences used as input. These shuffled sequences were then used as input to motif.freq.pl, producing output to allow exact comparison of the original sequence's frequency-bin distribution to the shuffled sequence's to assess for locations of motif enrichment or depletion. Shuffle.pl allows the user to specify the number of times that the motif shuffling is to be applied to the original input sequences. Thus, for a large number of shufflings and by averaging the shuffled frequencies in equivalent bins, a much lower 'noise' or variation in the shuffled random frequency-bin distribution can be achieved to compare to the original sequence motif bin distribution. As a test, real sequences were input into the shuffle.pl program to generate ten-fold randomly shuffled sequences. For a number of calculated short 
sequence motif frequency-bin distributions, these control shuffled distributions all exhibited frequency vs bin \# plots with low variation between bins. The linear fits to these plots possessed slopes near zero $\left(R^{2}<0.05\right)$, indicating no statistically significant trend in the shuffled sequential bin frequencies, as expected. In this study, we carried out 10 shufflings of each real sequence analyzed and used these as input to motif.freq.pl. The 10 motif.freq.pl output sequences' frequencies were then averaged for each bin to create an average random frequency bin distribution to compare to each real sequence. We call this frequency average of 10 shufflings: $F_{10 X}$ shuffle.

\section{Additional file}

Additional file 1: Supplementary Figures and Tables. Contains

Figures S1-S6 and Tables S1 and S2.

\section{Abbreviations}

EGASP: ENCODE Genome Annotation Assessment Project; fiNexp: Frequency (nucleotide $i$ and length $\mathrm{N}$ ) expected; fi $\mathrm{N}_{\mathrm{N}} \mathrm{Obs}$ : Frequency (nucleotide $\mathrm{i}$ and length $\mathrm{N}$ ) observed; $\mathrm{F}_{\text {obs: }}$ : Frequency observed; $\mathrm{F}_{10 \mathrm{x} \text { shuffle: Frequency }}$ following a 10-fold shuffle; FAIRE: Formaldehyde-assisted isolation of protein-free DNA; IGR: Intergenic regions; LCR: Low complexity region; MAINE: Micrococcal Nuclease-assisted isolation of nucleosome bound DNA; NDR: Nucleosome depleted region; $\mathrm{N}_{\max }$ exp: Maximum length of tract expected; $\mathrm{N}_{\max } \mathrm{Obs}$ : Maximum length of tract observed; ORF: Open reading frame; P: Proportion; PIC: Pre-initiation complex; R: Representation; RNAPollI: RNA polymerase II complex; Type A IGR: An IGR flanked with head-to-head genes containing two promoters; Type C IGR: An IGR flanked with tail-to-tail genes containing two terminators.

\section{Competing interests}

The authors declare that they have no competing interests.

\section{Authors' contributions}

KR helped design the study, carried out the majority of the bioinformatics analyses described and drafted the original manuscript. CHC participated in the design, writing and testing of the motif.freq.pl and shuffle.pl programs. JWB contributed the program Poly to this study, and also participated in analyzing data. NP and KLR participated in the design and execution of the nucleosome positioning analysis, providing MAINE/FAIRE data and sequences around predicted core promoters. RDE designed and wrote algorithms to secure the sequences used for all species used in this study and provided support in the analysis of data. KAM helped create Poly, motif. freq.pl and shuffle.pl and participated in the study design and its coordination and revised and helped finalize the manuscript in all its revisions. PH designed the study, coordinated the research, assisted in data analysis and produced the final version of the manuscript. All authors read and approved the final manuscript.

\section{Acknowledgements}

We would also like to thank Catherine Merrick who provided extensive feedback during the preparation of the manuscript. This work was supported by a Biotechnology \& Biological Sciences Research Council (BBSRC, BB/ H002405/1) New Investigator Award to PH and BBSRC PhD award to KR.

\section{Author details}

'Institute for Science and Technology in Medicine, Keele University, Stoke-on-Trent ST5 5BG, Staffordshire, UK. ${ }^{2}$ Center for Intelligent Biomaterials, University of Massachusetts Lowell, Lowell, MA 01854, USA. ${ }^{3}$ Bioinformatics Organization Inc, Hudson, MA 01749, USA. ${ }^{4}$ National Institute for Agricultural Research (INRA), UR1264-Mycology and Food Safety (MycSA), CS20032, 33882 Villenave d'Ornon Cedex, France. ${ }^{5}$ School of Veterinary Medicine and Science, University of Nottingham, Nottingham LE12 5RD, Leicestershire, UK.

${ }^{6}$ Advanced Data Analysis Centre, University of Nottingham, Nottingham, UK.
${ }^{7}$ Department Cell Biology and Neuroscience, University of California, Riverside, CA 92521, USA. ${ }^{8}$ Currently at Institute for Aging Research, Hebrew SeniorLife, Boston, MA 02131, USA.

Received: 21 May 2014 Accepted: 24 September 2014

Published: 3 October 2014

\section{References}

1. Dechering KJ, Cuelenaere K, Konings RN, Leunissen JA: Distinct frequency-distributions of homopolymeric DNA tracts in different genomes. Nucl Acids Res 1998, 26:4056-4062.

2. Marx KA, Zhou Y, Kishawi IQ: Evidence for long poly(dA).poly(dT) tracts in $D$. discoideum DNA at high frequencies and their preferential avoidance of nucleosomal DNA core regions. J Biomol Struct Dyn 2006, 23:429-446.

3. Zhou Y, Bizzaro JW, Marx KA: Homopolymer tract length dependent enrichments in functional regions of 27 eukaryotes and their novel dependence on the organism DNA $(\mathrm{G}+\mathrm{C}) \%$ composition. BMC Genomics 2004, 5:95-104.

4. Field $Y$, Kaplan N, Fondufe-Mittendorf $Y$, Moore IK, Sharon E, Lubling $Y$, Widom J, Segal E: Distinct modes of regulation by chromatin encoded through nucleosome positioning signals. PLoS Comp Biol 2008, 4: e1000216.

5. Mavrich TN, loshikhes IP, Venters BJ, Jiang C, Tomsho LP, Qi J, Schuster SC, Albert I, Pugh BF: A barrier nucleosome model for statistical positioning of nucleosomes throughout the yeast genome. Genome Res 2008, 18:1073-1083

6. Dai J, Chuang RY, Kelly TJ: DNA replication origins in the Schizosaccharomyces pombe genome. Proc Natl Acad Sci U S A 2005, 102:337-342.

7. Segal E, Widom J: Poly(dA:dT) tracts: major determinants of nucleosome organization. Curr Opin Struct Biol 2009, 19:65-71.

8. Cohanim AB, Haran TE: The coexistence of the nucleosome positioning code with the genetic code on eukaryotic genomes. Nucl Acids Res 2009, 37:6466-6476

9. Radman-Livaja M, Rando OJ: Nucleosome positioning: how is it established, and why does it matter? Dev Biol 2010, 339:258-266.

10. Segal E, Widom J: What controls nucleosome positions? Trends Genet 2009, 25:335-343.

11. Zhang Z, Wippo CJ, Wal M, Ward E, Korber P, Pugh BF: A packing mechanism for nucleosome organization reconstituted across a eukaryotic genome. Science 2011, 332:977-980.

12. Polson HEJ, Blackman MJ: A role for poly (dA) poly(dT) tracts in directing activity of the Plasmodium falciparum calmodulin gene promoter. Mol Biochem Parasitol 2005, 141:179-189.

13. Porter ME: Positive and negative effects of deletions and mutation within the $5^{\prime}$ flanking sequences of Plasmodium falciparum DNA polymerase delta. Mol Biochem Parasitol 2002, 122:9-19.

14. Ponts N, Harris EY, Prudhomme J, Wick I, Eckhardt-Ludka C, Hicks GR, Hardiman G, Lonardi S, Le Roch KG: Nucleosome landscape and control of transcription in the human malaria parasite. Genome Res 2010, 20:228-238.

15. Westenberger SJ, Cui L, Dharia N, Winzeler E, Cui L: Genome-wide nucleosome mapping of Plasmodium falciparum reveals histone-rich coding and histone-poor intergenic regions and chromatin remodeling of core and subtelomeric genes. BMC Genomics 2009, 10:610-621.

16. Bunnik EM, Polishko A, Prudhomme J, Ponts N, Gill SS, Lonardi S, Le Roch KG: DNA-encoded nucleosome occupancy is associated with transcription levels in the human malaria parasite Plasmodium falciparum. BMC Genomics 2014, 15:347-353.

17. Cui L, Miao J: Chromatin-mediated epigenetic regulation in the malaria parasite Plasmodium falciparum. Euk Cell 2010, 9:1138-1149.

18. Duffy MF, Selvarajah SA, Josling GA, Petter M: The role of chromatin in Plasmodium gene expression. Cell Microbiol 2012, 14:819-828.

19. Ay F, Bunnik EM, Varoquaux N, Bol SM, Prudhomme J, Vert JP, Noble WS, Le Roch KG: Three-dimensional modeling of the $P$. falciparum genome during the erythrocytic cycle reveals a strong connection between genome architecture and gene expression. Genome Res 2014, 24:974-988.

20. Ponts N, Harris EY, Lonardi S, Le Roch KG: Nucleosome occupancy at transcription start sites in the human malaria parasite: a hard-wired evolution of virulence? Inf Gen Evol 2011, 11:716-724.

21. Horrocks $P$, Wong E, Russell K, Emes RD: Control of gene expression in Plasmodium falciparum - ten years on. Mol Biochem Parasitol 2009, 164:9-25. 
22. Russell K, Hasenkamp S, Emes R, Horrocks P: Analysis of the spatial and temporal arrangement of transcripts over intergenic regions in the human malarial parasite Plasmodium falciparum. BMC Genomics 2013, $14: 267-277$.

23. Siegel TN, Hon CC, Zhang Q, Lopez-Rubio JJ, Scheidig-Benatar C, Martins RM, Sismeiro O, Coppee JY, Scherf A: Strand-specific RNA-Seq reveals widespread and developmentally regulated transcription of natural antisense transcripts in Plasmodium falciparum. BMC Genomics 2014, 15:150-159.

24. Abrahamsen MS, Templeton TJ, Enomoto S, Abrahante JE, Zhu G, Lancto CA, Deng M, Liu C, Widmer G, Tzipori S, Buck GA, Xu P, Bankier AT, Dear PH, Konfortov BA, Spriggs HF, lyer L, Anantharaman V, Aravind L, Kapur V: Complete genome sequence of the apicomplexan Cryptosporidium parvum. Science 2004, 304:441-445.

25. Brayton KA, Lau AO, Herndon DR, Hannick L, Kappmeyer LS, Berens SJ, Bidwell SL, Brown WC, Crabtree J, Fadrosh D, Feldblum T, Forberger HA, Haas BJ, Howell JM, Khouri H, Koo H, Mann DJ, Norimine J, Paulsen IT, Radune D, Ren Q, Smith RK Jr, Suarez CE, White O, Wortman JR, Knowles DP Jr, McElwain TF, Nene VM: Genome sequence of Babesia bovis and comparative analysis of apicomplexan hemoprotozoa. PLOS Path 2007 3:1401-1413.

26. Carlton JM, Adams JH, Silva JC, Bidwell SL, Lorenzi H, Caler E, Crabtree J, Angiuoli SV, Merino EF, Amedeo P, Cheng Q, Coulson RM, Crabb BS, Del Portillo HA, Essien K, Feldblyum TV, Fernandez-Becerra C, Gilson PR, Gueye AH, Guo X, Kang'a S, Kooij TW, Korsinczky M, Meyer EV, Nene V, Paulsen I, White O, Ralph SA, Ren Q, Sargeant TJ, et al: Comparative genomics of the neglected human malaria parasite Plasmodium vivax. Nature 2008 455:757-763.

27. Carlton JM, Adams JH, Silva JC, Bidwell SL, Lorenzi H, Caler E, Crabtree J, Angiuoli SV, Merino EF, Amedeo P, Cheng Q, Coulson RM, Crabb BS, Del Portillo HA, Essien K, Feldblyum TV, Fernandez-Becerra C, Gilson PR, Gueye AH, Guo X, Kang'a S, Kooij TW, Korsinczky M, Meyer EV, Nene V, Paulsen I, White O, Ralph SA, Ren Q, Sargeant TJ, et al: Genome sequence and comparative analysis of the model rodent malaria parasite Plasmodium yoelii yoelii. Nature 2002, 419:512-519.

28. Gardner MJ, Bishop R, Shah T, de Villiers EP, Carlton JM, Hall N, Ren Q, Paulsen IT, Pain A, Berriman M, Wilson RJ, Sato S, Ralph SA, Mann DJ, Xiong Z, Shallom SJ, Weidman J, Jiang L, Lynn J, Weaver B, Shoaibi A, Domingo AR, Wasawo D, Crabtree J, Wortman JR, Haas B, Angiuoli SV, Creasy TH, Lu C, Suh B, et al: Genome sequence of Theileria parva, a bovine pathogen that transforms lymphocytes. Science 2005, 309:134-137.

29. Gardner MJ, Hall N, Fung E, White O, Berriman M, Hyman RW, Carlton JM, Pain A, Nelson KE, Bowman S, Paulsen IT, James K, Eisen JA, Rutherford K, Salzberg SL, Craig A, Kyes S, Chan MS, Nene V, Shallom SJ, Suh B, Peterson J, Angiuoli S, Pertea M, Allen J, Selengut J, Haft D, Mather MW, Vaidya AB, Martin DM, et al: Genome sequence of the human malaria parasite Plasmodium falciparum. Nature 2002, 419:498-511.

30. Pain A, Bohme U, Berry AE, Mungall K, Finn RD, Jackson AP, Mourier T, Mistry J, Pasini EM, Aslett MA, Balasubrammaniam S, Borgwardt K, Brooks K Carret C, Carver TJ, Cherevach I, Chillingworth T, Clark TG, Galinski MR, Hall N, Harper D, Harris D, Hauser H, Ivens A, Janssen CS, Keane T, Larke N, Lapp S, Marti M, Moule S, et al: The genome of the simian and human malaria parasite Plasmodium knowlesi. Nature 2008, 455:799-803.

31. Pain A, Böhme U, Berry AE, Mungall K, Finn RD, Jackson AP, Mourier T, Mistry J, Pasini EM, Aslett MA, Balasubrammaniam S, Borgwardt K, Brooks K, Carret C, Carver TJ, Cherevach I, Chillingworth T, Clark TG, Galinski MR, Hall N, Harper D, Harris D, Hauser H, Ivens A, Janssen CS, Keane T, Larke N, Lapp S, Marti M, Moule $S$, et al: Genome of the host-cell transforming parasite Theileria annulata compared with T. parva. Science 2005, 309:131-133.

32. Reid AJ, Vermont SJ, Cotton JA, Harris D, Hill-Cawthorne GA, Könen-Waisman S, Latham SM, Mourier T, Norton R, Quail MA, Sanders M, Shanmugam D, Sohal A, Wasmuth JD, Brunk B, Grigg ME, Howard JC, Parkinson J, Roos DS, Trees AJ, Berriman M, Pain A, Wastling JM: Comparative genomics of the apicomplexan parasites Toxoplasma gondii and Neospora caninum: Coccidia differing in host range and transmission strategy. PLOS Path 2012, 8:e1002567.

33. Xu P, Widmer G, Wang Y, Ozaki LS, Alves JM, Serrano MG, Puiu D, Manque P. Akiyoshi D, Mackey AJ, Pearson WR, Dear PH, Bankier AT, Peterson DL, Abrahamsen MS, Kapur V, Tzipori S, Buck GA: The genome of Cryptosporidium hominis. Nature 2004, 431:1107-1112.

34. Bizzaro JW, Marx KA: Poly: a quantitative analysis tool for simple sequence repeat (SSR) tracts in DNA. BMC Bioinformatics 2003, 4:22-25.
35. Brick K, Watanabe J, Pizzi E: Core promoters are predicted by their distinct physicochemical properties in the genome of Plasmodium falciparum. Genome Biol 2008, 9(12):178-184.

36. Denver DR, Morris K, Kewalramani A, Harris KE, Chow A, Estes S, Lynch M, Thomas WK: Abundance, distribution, and mutation rates of homopolymeric nucleotide runs in the genome of Caenorhabditis elegans. J Mol Evol 2004, 58:584-595.

37. Katti MV, Ranjekar PK, Gupta VS: Differential distribution of simple sequence repeats in eukaryotic genome sequences. Mol Biol Evol 2001, 18:1161-1167.

38. Toth G, Gaspari Z, Jurka J: Microsatellites in different eukaryotic genomes: survey and analysis. Genome Res 2000, 10:967-981.

39. Hancock JM: The contribution of slippage-like processes to genome evolution. J Mol Evol 1995, 41:1038-1047.

40. Nadir E, Margalit H, Gallily T, Ben-Sasson SA: Microsatellite spreading in the human genome: evolutionary mechanisms and structural implications. Proc Natl Acad Sci U S A 1996, 93:6470-6475.

41. Tautz D, Trick M, Dover GA: Cryptic simplicity in DNA is a major source of genetic variation. Nature 1986, 322:652-656.

42. Wilder J, Hollocher $\mathrm{H}$ : Mobile elements and the genesis of microsatellites in dipterans. Mol Biol Evol 2001, 18:384-392.

43. DeBarry JD, Kissinger JC: Jumbled genomes: missing Apicomplexan synteny. Mol Biol Evol 2011, 28:2855-2871.

44. Durand PM, Oelofse AJ, Coetzer TL: An analysis of mobile genetic elements in three Plasmodium species and their potential impact on the nucleotide composition of the $P$. falciparum genome. BMC Genomics 2006, 7:282-287.

45. Roy SW, Hartl DL: Very little intron loss/gain in Plasmodium: intron loss/ gain mutation rates and intron number. Genome Res 2006, 16:750-756.

46. Roy SW, Penny D: Large-scale intron conservation and order-ofmagnitude variation in intron loss/gain rates in apicomplexan evolution. Genome Res 2006, 16:1270-1275.

47. Chang GS, Noegel AA, Mavrich TN, Müller R, Tomsho L, Ward E, Felder M, Jiang C, Eichinger L, Glockner G, Glöckner G, Schuster SC, Pugh BF: Unusual combinatorial involvement of poly-A/T tracts in organizing genes and chromatin in Dictyostelium. Genome Res 2012, 22:1098-1106.

48. Sims JS, Militello KT, Sims PA, Patel VP, Kasper JM, Wirth DF: Patterns of genespecific and total transcriptional activity during the Plasmodium falciparum intraerythrocytic developmental cycle. Euk Cell 2009, 8(3):327-338.

49. Gopalakrishnan AM, Nyindodo LA, Ross Fergus M, Lopez-Estrano C: Plasmodium falciparum: preinitiation complex occupancy of active and inactive promoters during erythrocytic stage. Exp Parasitol 2009, 121:46-54.

50. Kishore SP, Perkins SL, Templeton TJ, Deitsch KW: An unusual recent expansion of the C-terminal domain of RNA polymerase II in primate malaria parasites features a motif otherwise found only in mammalian polymerases. J Mol Evol 2009, 68:706-714

51. Bartfai $R$, Hoeijmakers WA, Salcedo-Amaya AM, Smits AH, Janssen-Megens $E$ Kaan A, Treeck M, Gilberger TW, Francoijs KJ, Stunnenberg HG: H2A.Z demarcates intergenic regions of the Plasmodium falciparum epigenome that are dynamically marked by H3K9ac and H3K4me3. PLoS Path 2010, 6(12):e1001223.

52. Depledge DP, Lower RP, Smith DF: RepSeq-a database of amino acid repeats present in lower eukaryotic pathogens. BMC Bioinformatics 2007, 8:122-127.

53. Frugier M, Bour T, Ayach M, Santos MA, Rudinger-Thirion J, Theobald-Dietrich A, Pizzi E: Low Complexity Regions behave as tRNA sponges to help co-translational folding of plasmodial proteins. FEBS Lett 2010, 584:448-454

54. Pizzi E, Frontali C: Divergence of noncoding sequences and of insertions encoding nonglobular domains at a genomic region well conserved in plasmodia. J Mol Evol 2000, 50:474-480

55. Pizzi E, Frontali C: Low-complexity regions in Plasmodium falciparum proteins. Genome Res 2001, 11:218-229.

56. Zilversmit MM, Volkman SK, DePristo MA, Wirth DF, Awadalla P, Hartl DL: Low-complexity regions in Plasmodium falciparum: missing links in the evolution of an extreme genome. Mol Biol Evol 2010, 27:2198-2209.

57. Carter R, Nijhout MM: Control of gamete formation (exflagellation) in malaria parasites. Science 1977, 195:407-409.

58. Janse CJ, van der Klooster PF, van der Kaay HJ, van der Ploeg M, Overdulve JP: DNA synthesis in Plasmodium berghei during asexual and sexual development. Mol Biochem Parasitol 1986, 20:173-182. 
59. Janse CJ, Van der Klooster PF, Van der Kaay HJ, Van der Ploeg M, Overdulve JP: Rapid repeated DNA replication during microgametogenesis and DNA synthesis in young zygotes of Plasmodium berghei. Trans Roy Soc Trop Med Hyg 1986, 80:154-157.

60. Schmitt MW, Venkatesan RN, Pillaire MJ, Hoffmann JS, Sidorova JM, Loeb LA: Active site mutations in mammalian DNA polymerase delta alter accuracy and replication fork progression. J Biol Chem 2010, 285:32264-32272.

doi:10.1186/1471-2164-15-848

Cite this article as: Russell et al:: Homopolymer tract organization in the human malarial parasite Plasmodium falciparum and related Apicomplexan parasites. BMC Genomics 2014 15:848.

\section{Submit your next manuscript to BioMed Central and take full advantage of:}

- Convenient online submission

- Thorough peer review

- No space constraints or color figure charges

- Immediate publication on acceptance

- Inclusion in PubMed, CAS, Scopus and Google Scholar

- Research which is freely available for redistribution 